\title{
Different Classical Methods of Estimation and Chi-squared Goodness-of-fit Test for Unit Generalized Inverse Weibull Distribution
}

\author{
Aidi khaoula \\ University Badji \\ Mokhtar
}

\author{
Sanku Dey \\ St. Anthony's College
}

\author{
Devendra Kumar \\ Central University \\ of Haryana
}

\author{
Seddik-Ameur N \\ University Badji \\ Mokhtar
}

\begin{abstract}
In this paper, we try to contribute to the distribution theory literature by incorporating a new bounded distribution, called the unit generalized inverse Weibull distribution (UGIWD) in the $(0,1)$ intervals by transformation method. The proposed distribution exhibits increasing and bathtub shaped hazard rate function. We derive some basic statistical properties of the new distribution. Based on complete sample, the model parameters are obtained by the methods of maximum likelihood, least square, weighted least square, percentile, maximum product of spacing and Cramèr-von-Mises and compared them using Monte Carlo simulation study. In addition, bootstrap confidence intervals of the parameters of the model based on aforementioned methods of estimation are also obtained. We illustrate the performance of the proposed distribution by means of one real data set and the data set shows that the new distribution is more appropriate as compared to unit Birnbaum-Saunders, unit gamma, unit Weibull, Kumaraswamy and unit Burr III distributions. Further, we construct chi-squared goodness-of-fit tests for the UGIWD using right censored data based on Nikulin-Rao-Robson (NRR) statistic and its modification. The criterion test used is the modified chi-squared statistic $Y^{2}$, developed by Bagdonavičius and Nikulin (2011) for some parametric models when data are censored. The performances of the proposed test are shown by an intensive simulation study and an application to real data set
\end{abstract}

Keywords: chi-squared test, Cramèr-von-Mises estimators, maximum likelihood estimators, maximum product of spacing estimators, right censored data.

\section{Introduction}

An integral part of many statistical studies is the collection of information about the form of population from which the data is obtained. For this purpose, statisticians often use goodness of fit (GOF) tests so as to determine whether the observed sample data "fits" some proposed model. To validate the model, tests such as graphical tests, chi-squared tests, KolmogorovSmirnov statistic, Anderson-Darling statistic and many others are employed. The objective of these tests is to measure the distance between the observed values and the expected theoretical values. The chosen (or selected) model will be rejected when this distance is found to be 
greater than the critical value. The standard tables for these tests are considered to be invalid when the parameters are unknown. Further, goodness of fit tests for complete sample procedures are inappropriate in case of censored samples (see Badr (2019)).

We find many useful studies on GOF in statistical literature, especially, when the model is well specified. Readers may refer to the works of: Stephens (1970), Stephens (1974), Durbin (1975), Green and Hegazy (1976), Chandra, Singpurwalla, and Stephens (1981), Murthy, Xie, and Jiang (2004), Abdelfattah (2008), Yen and Moore (1988), Balakrishnan and Basu (1995), Hassan (2005), Abd-Elfattah (2011), Wang (2008), Al-Omari and Zamanzade (2016), Aidi and Seddik-Ameur (2016), Zamanzade and Mohdizadeh (2017), Goual and Yousof (2020) and many others. It is to be noted that when we consider censored data and the parameters of the model are unknown as in case of reliability and medical studies, the research problem remains open as much needs to be investigated as regards to adequacy of newly introduced distributions.

Of late, several studies have been carried out to define new families of inverse Weibull distribution, such as De Gusmao, Ortega, and Cordeiro (2011) proposed the generalized inverse Weibull distribution, Khan and King (2012) proposed modified inverse Weibull distribution, Hanook, Shahbaz, Mohsin, and Kibria (2013) introduced beta inverse Weibull distribution, Pararai, Warahena, and Oluyede (2014) proposed gamma inverse Weibull distribution, Aryal and Elbatal (2015) introduced Kumaraswamy modified inverse Weibull distribution, Elbatal, Condino, and Domma (2016) proposed reflected generalized beta inverse Weibull distribution, Okasha, El-Baz, Tarabia, and Basheer (2017) introduced Marshall-Olkin extended inverse Weibull distribution, Mudasir and Ahmad (2018) introduced weighted version of generalized inverse Weibull distribution and Basheer (2019) introduced alpha power inverse Weibull distribution and the reference cited therein.

The above cited distributions are extended form of inverse Weibull distribution and have been derived by incorporating some additional parameters to the original probability distribution. In addition, they are based on the support over positive part of the real line. At the same time, probability distributions with support on finite range play a key role in many studies. For instance, many life test experiments which cater to data on some finite range, such as data on fractions, percentages, per capita income growth, fuel efficiency of vehicles, height and weight of individuals, survival times from a deadly disease etc. are likely to lie in some bounded positive intervals (see Kumaraswamy (1980), Gomez-Deniz, Sordo, and Caldern-Ojeda (2013), Mazucheli, Menezes, and Ghitany (2018a), Mazucheli, Menezes, and Dey (2018b), Mazucheli, Menezes, and Dey (2018c), Mazucheli, Menezes, and Dey (2019)). Due to evolving problems in life testing experiments, statistician require more and more distributions with finite support.

In this paper, first we derive a new bounded distribution from the generalized inverse Weibull distribution by transformation of the type $x=\frac{T}{1+T}$, where $T$ has the generalized inverse Weibull distribution. We obtain a new distribution with support on $(0,1)$, which we refer to as unit generalized inverse Weibull distribution (UGIWD). This distribution is capable of modelling increasing and bathtub shaped hazard rate. Second, we obtain maximum likelihood, least square, weighted least square, percentile, maximum product of spacing and Cramèr-von-Mises estimators for the unknown parameters of the model based on complete sample. Besides, bootstrap confidence intervals (BCIs) of the parameters of the model based on above cited methods of estimation are also obtained. Next, we construct chi-squared tests for the UGIWD when data are right censored. We use modified chi-squared statistic developed by Bagdonavičius, Levuliene, and Nikulin (2013) for some parametric accelerated failure times models. This technique has been used to validate some models like, Weibull extension accelerated failure time model (Seddik-Ameur and Wafa 2018), competing risk model (Chouia and Seddik-Ameur 2017).

The organization of this article is as follows: In Section 2, model description is provided. In Section 3, some basic properties of the model are derived. In Section 4, six different classical methods of estimation based on complete samples are discussed. Monte Carlo simulation 
study is carried out to compare the different methods of estimation in Section 5. The potentiality of the new model is illustrated by means of an application to real data set in Section 6. In Section 7, maximum likelihood estimates based on right censored data is discussed. Estimated Fisher information matrix is obtained in Section 8. In Section 9, test statistic for right censored data is proposed for the model. In order to study the performance of the test statistic, a simulation study is carried out based on right censored samples in Section 10. In order to confirm the practicability of the proposed goodness-of-fit test, and the usefulness of this model, one real data set is analyzed in Section 11. At the end of this paper, conclusions are given in Section 12 .

\section{Model description}

If a random variable $\mathrm{T}$ follows generalized inverse Weibull (GIW) distribution, then $X=$ $\frac{T}{1+T}$ follows a $U G I W D$. The cumulative distribution function of generalized inverse Weibull (GIW) distribution is given by

$$
F(t)=e^{-\alpha(\lambda / t)^{\beta}}, \quad t>0, \alpha, \beta, \lambda>0 .
$$

Thus the UGIWD with three parameters has the density function

$$
f(x)=\frac{\alpha \lambda \beta}{x^{2}}\left(\frac{\lambda(1-x)}{x}\right)^{\beta-1} \exp \left\{-\alpha\left(\frac{\lambda(1-x)}{x}\right)^{\beta}\right\}, \quad 0<x<1, \alpha, \beta, \lambda>0 .
$$

The cumulative distribution function (CDF), survival function (SF) and hazard rate function of UGIWD are, respectively given by

$$
\begin{gathered}
F(x)=\exp \left\{-\alpha\left(\frac{\lambda(1-x)}{x}\right)^{\beta}\right\}, \\
S(x)=1-\exp \left\{-\alpha\left(\frac{\lambda(1-x)}{x}\right)^{\beta}\right\}, \\
h(x)=\frac{\frac{\alpha \lambda \beta}{x^{2}}\left(\frac{\lambda(1-x)}{x}\right)^{\beta-1} \exp \left\{-\alpha\left(\frac{\lambda(1-x)}{x}\right)^{\beta}\right\}}{1-\exp \left\{-\alpha\left(\frac{\lambda(1-x)}{x}\right)^{\beta}\right\}} .
\end{gathered}
$$

and the cumulative hazard rate function is given by

$$
H(x)=-\ln S(t)=-\ln \left(1-\exp \left\{-\alpha\left(\frac{\lambda(1-x)}{x}\right)^{\beta}\right\}\right)
$$

For some parameter values, Figures 1 indicate the probability density function, hazard func- 

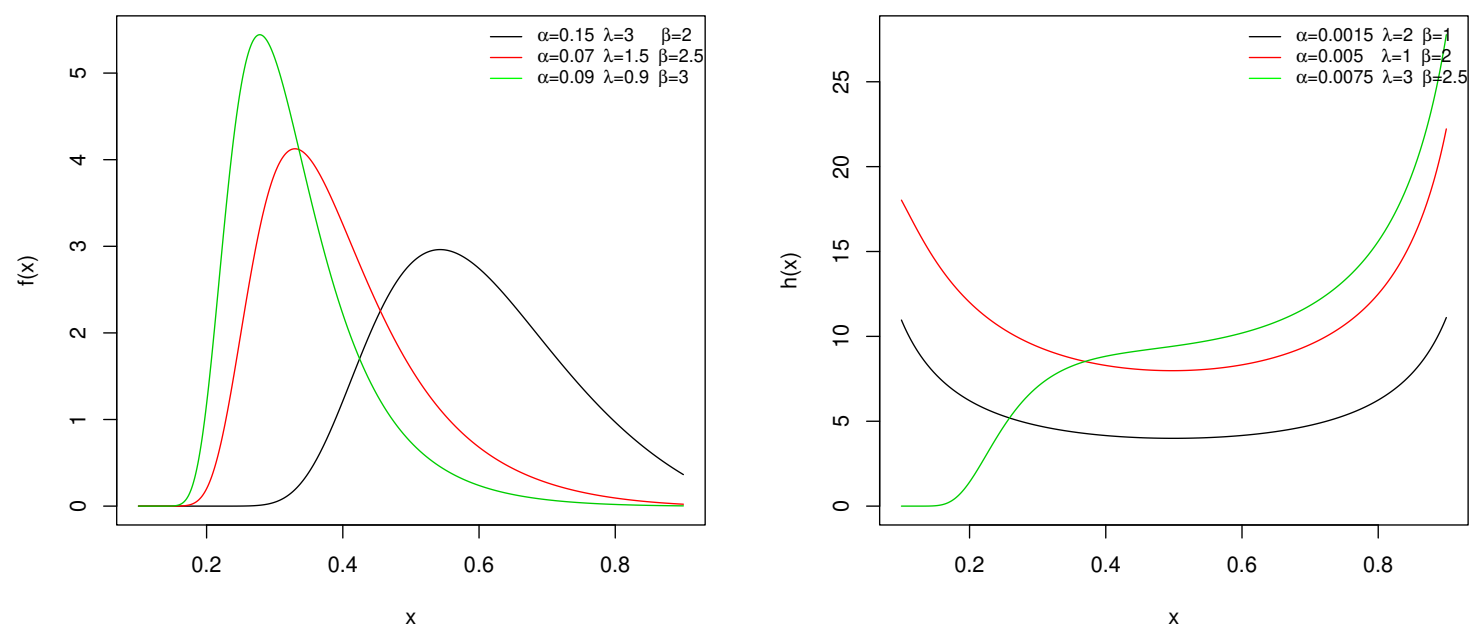

Figure 1: Probability density function and hazard function of the UGIW

\section{Statistical and mathematical properties}

In this section, we devoted to some statistical and mathematical properties of the UGIW distribution.

\subsection{Moments and moment generating function}

The moments, incomplete moments, moment generating function, skewness and kurtosis of a probability distribution are very important tools to illustrate the distribution. The nth moments of the UGIW distribution is given by

$$
\begin{aligned}
E\left(X^{n}\right) & =\int_{0}^{\infty} x^{n} f(x) d x=\alpha \lambda \beta \int_{0}^{1} x^{n-2}\left(\frac{\lambda(1-x)}{x}\right)^{\beta-1} \exp \left[-\alpha\left(\frac{\lambda(1-x)}{x}\right)^{\beta}\right] d x \\
& =\alpha \lambda^{n} \beta \int_{0}^{\infty}(\lambda+t)^{-n} t^{\beta-1} e^{-\alpha t^{\beta}} d t
\end{aligned}
$$

where $t=\left(\frac{\lambda(1-x)}{x}\right)$. For $|x|<\lambda$ and negative integer $-n$, the power series holds

$$
(x+\lambda)^{-n}=\sum_{i=0}^{\infty}(-1)^{i}\left(\begin{array}{c}
n+i-1 \\
i
\end{array}\right) x^{i} \lambda^{-i-n} .
$$

Hence, we can write

$$
\begin{aligned}
E\left(X^{n}\right) & =\alpha \lambda^{-i} \beta \sum_{i=0}^{\infty}(-1)^{i}\left(\begin{array}{c}
n+i-1 \\
i
\end{array}\right) \int_{0}^{\infty} t^{\beta+i-1} e^{-\alpha t^{\beta}} d t \\
& =\sum_{i=0}^{\infty}(-1)^{i}\left(\begin{array}{c}
n+i-1 \\
i
\end{array}\right) \lambda^{-i} \alpha^{-\frac{i}{\beta}} \Gamma\left(\frac{i}{\beta}+1\right) .
\end{aligned}
$$

The moment generating function of the UGIW distribution can be computed as

$$
M_{x}(t)=\sum_{p=0}^{\infty} \sum_{i=0}^{\infty}(-1)^{i}\left(\begin{array}{c}
p+i-1 \\
i
\end{array}\right) \frac{t^{p} \lambda^{-i} \alpha^{-\frac{i}{\beta}}}{p !} \Gamma\left(\frac{i}{\beta}+1\right) .
$$


In Table 1, we have presented the expected values, variances, skewness and kurtosis of the UGIW distribution for various values of $\alpha$ and $\beta$. One can see from Table 1 that the the means and variances are increasing with respect to $\alpha$ and $\beta$ but covariance (CV), skewness and kurtosis are decreasing with respect to $\alpha$ and $\beta$.

Table 1: Moment calculations for various values of $\alpha$ and $\beta$ when $\lambda=1$

\begin{tabular}{rrlrlrr}
\hline$\alpha$ & $\beta$ & Mean & Variance & CV & Skewness & Kurtosis \\
\hline 0.5 & 0.5 & 0.24381 & 0.28025 & 0.76775 & 1.52505 & 8.30275 \\
& 1 & 0.41725 & 0.41185 & 0.54382 & 1.14310 & 5.43955 \\
& 5 & 1.03315 & 0.63835 & 0.27341 & 0.74905 & 3.39335 \\
& 10 & 1.35185 & 0.68225 & 0.21602 & 0.69142 & 3.17452 \\
& 15 & 1.68401 & 0.70605 & 0.17641 & 0.66181 & 3.07025 \\
& 35 & 2.02342 & 0.71845 & 0.14810 & 0.64682 & 3.01955 \\
1.5 & 0.5 & 0.34713 & 0.39945 & 0.64375 & 1.21445 & 5.79085 \\
& 1 & 0.55171 & 0.54950 & 0.47505 & 0.92840 & 4.09061 \\
& 5 & 1.20785 & 0.77995 & 0.25850 & 0.62802 & 2.84415 \\
& 10 & 1.53302 & 0.82181 & 0.20905 & 0.58331 & 2.70830 \\
& 15 & 1.86855 & 0.84423 & 0.17385 & 0.56023 & 2.64335 \\
& 35 & 2.20955 & 0.85585 & 0.14805 & 0.54851 & 2.61175 \\
5 & 0.5 & 0.47515 & 0.52135 & 0.53725 & 0.98580 & 4.35995 \\
& 1 & 0.71141 & 0.67335 & 0.40781 & 0.76565 & 3.31075 \\
& 5 & 1.40605 & 0.88215 & 0.23615 & 0.53295 & 2.53915 \\
& 10 & 1.73710 & 0.91775 & 0.19503 & 0.49805 & 2.45535 \\
& 15 & 2.07583 & 0.93661 & 0.16485 & 0.48429 & 2.41541 \\
& 35 & 2.41835 & 0.94630 & 0.14221 & 0.47081 & 2.39595 \\
10 & 0.5 & 0.55005 & 0.57881 & 0.48904 & 0.89450 & 3.88942 \\
& 1 & 0.80203 & 0.72430 & 0.37515 & 0.70195 & 3.06553 \\
& 5 & 1.51485 & 0.91262 & 0.22295 & 0.49901 & 2.46351 \\
& 10 & 1.84870 & 0.94365 & 0.18582 & 0.46862 & 2.39865 \\
15 & 2.18875 & 0.95995 & 0.15825 & 0.45285 & 2.36785 \\
& 35 & 2.53205 & 0.96835 & 0.13742 & 0.44485 & 2.35285 \\
15 & 0.5 & 0.62272 & 0.62515 & 0.44890 & 0.82570 & 3.57405 \\
& 1 & 0.88815 & 0.76061 & 0.34715 & 0.65605 & 2.91260 \\
& 5 & 1.61610 & 0.92652 & 0.21055 & 0.47885 & 2.43381 \\
& 10 & 1.95221 & 0.95295 & 0.17682 & 0.45241 & 2.38272 \\
& 15 & 2.29343 & 0.96680 & 0.15163 & 0.43875 & 2.35851 \\
35 & 0.5 & 0.69151 & 0.66115 & 0.41575 & 0.77440 & 3.36180 \\
& 1 & 0.96825 & 0.78495 & 0.32351 & 0.62435 & 2.82031 \\
& 5 & 1.70842 & 0.92891 & 0.19945 & 0.46981 & 2.43223 \\
& 10 & 2.04625 & 0.95120 & 0.16850 & 0.44695 & 2.39105 \\
& 15 & 2.38840 & 0.96281 & 0.14525 & 0.43515 & 2.37155 \\
& 35 & 2.73275 & 0.96873 & 0.12735 & 0.42915 & 2.36210 \\
\hline & & & & & &
\end{tabular}

\subsection{Quantiles, L-moments and measures of skewness and kurtosis}

The characteristics of probability distribution are also measured by the quantile function like the moments. Also, the quantile function represents the distribution and can be considered as an alternative tool for data analysis, see Nair, Sankaran, and Balakrishnan (2013). Let $F\left(Q_{p} ; \alpha, \lambda, \beta\right)$ be the CDF of the UGIW distribution at pth quantiles $Q_{p}$. Then the pth 
quantile of the UGIW random variable is given by

$$
x_{p}=\left\{\frac{1}{\lambda}\left(-\frac{\ln p}{\alpha}\right)^{1 / \beta}+1\right\}^{-1} .
$$

In particular, the first three quantiles, $Q_{1}, Q_{2}$ and $Q_{3}$, can be obtained by setting $p=0.25$, $p=0.5$ and $p=0.75$ in equation (7), respectively.

An application of quantile function is the L-moments. L-moments are the linear combinations of order statistics and can be used to compute the mean, standard deviation, skewness and kurtosis of the distribution. The sth L-moment is defined by

$$
L_{s}=\int_{0}^{1} \sum_{i=0}^{s-1}(-1)^{s-i-1}\left(\begin{array}{c}
s-1 \\
i
\end{array}\right)\left(\begin{array}{c}
s-1+i \\
i
\end{array}\right) u^{i} Q(u) d u .
$$

The coefficient of skewness and kurtosis based on quantiles are given by

$$
\text { (Galton cofficient) } S=\frac{Q_{0.25}+Q_{0.75}-2 Q_{0.5}}{Q_{0.75}-Q_{0.25}}
$$

and

$$
\text { (Moors cofficient) } T=\frac{Q_{0.875}-Q_{0.625}+Q_{0.375}-Q_{0.125}}{Q_{0.75}-Q_{0.25}} .
$$

\subsection{Conditional moment and mean deviation}

Here, we introduce an important lemma which will be used in the next section.

Lemma 1. Let $X$ be a random variable with pdf given in (1) and let $J_{n}(t)=\int_{0}^{t} x^{n} f(x) d x$. Then we have

$$
J_{n}(t)=\lambda^{n} \sum_{i=0}^{\infty}(-1)^{i}\left(\begin{array}{c}
n+i-1 \\
i
\end{array}\right) \alpha^{-\frac{i}{\beta}-1} \gamma\left(\frac{i}{\beta}+1, \beta\left(\frac{\lambda(1-t)}{t}\right)^{\beta}\right),
$$

where $\gamma(a, x)$ denote the incomplete gamma function and defined by $\gamma(a, x)=\int_{0}^{x} t^{a-1} e^{-t} d t$. Proof. Using equation (1), we have

$$
\begin{aligned}
J_{n}(t) & =\int_{0}^{t} x^{n} f(x) d x=\alpha \lambda \beta \int_{0}^{t} x^{n-2}\left(\frac{\lambda(1-x)}{x}\right)^{\beta-1} \exp \left[-\alpha\left(\frac{\lambda(1-x)}{x}\right)^{\beta}\right] d x \\
& =\alpha \lambda^{n} \beta \sum_{i=0}^{\infty}(-1)^{i}\left(\begin{array}{c}
n+i-1 \\
i
\end{array}\right) \int_{0}^{\frac{\lambda(1-t)}{t}} u^{\beta+i-1} e^{-\alpha u^{\beta}} d u,
\end{aligned}
$$

where $u=\left(\frac{\lambda(1-x)}{x}\right)$. The result follows by using Equation (3.381.8) page 346 in Gradshteyn and Ryzhik (2014) to calculate the integral in (9). The proof is complete.

The $n$-th conditional moments of the UGIW distribution is given by

$$
\eta_{n}(t)=E\left[X^{n} \mid x>t\right]=\frac{1}{1-F(t)} \int_{t}^{\infty} x^{n} f(x) d x=\frac{1}{S(t)}\left[E\left(X^{n}\right)-J_{n}(t)\right] .
$$

It can be expressed by using (3), (6) and (8). The same remark holds for the $n$-th reversed moments of the UGIW distribution and is given by

$$
m_{n}(t)=E\left[X^{n} \mid x \leq t\right]=\frac{1}{F(t)} \int_{0}^{t} x^{n} f(x) d x=\frac{1}{F(t)} J_{n}(t) .
$$


An application of the conditional moments is the mean residual life (MRL). MRL function is the expected remaining life, $X-x$, given that the item has survived to time $x$. Thus, in life testing situations, the expected additional lifetime given that a component has survived until time $x$ is called the (MRL). The MRL function in terms of the first conditional moment as

$$
m_{X}(x)=E(X-x \mid X>x)=\frac{1}{S(x)} J_{1}(x)-x .
$$

where $J_{1}(x)$ can be obtained from (9) when $n=1$.

Another application of the conditional moments is the mean deviations about the mean and the median. They are used to measure the dispersion and the spread in a population from the center. If we denote the median by $M$, then the mean deviations from the mean and the median can be calculated as

$$
\delta_{\mu}=\int_{0}^{\infty}|x-\mu| f(x) d x=2 \mu F(\mu)-2 \mu+2 J_{1}(\mu)
$$

and

$$
\delta_{M}=\int_{0}^{\infty}|x-M| f(x) d x=2 J_{1}(M)-\mu
$$

respectively. Where $J_{1}(\mu)$ and $J_{1}(M)$ can obtained from (8). Also, $F(\mu)$ and $F(M)$ can be easily calculated from (2).

\subsection{Entropy and stress-strength reliability}

Entropy is useful in gathering information about the uncertainty of the random experiment. It was initially used in assessing the quality of communications. The Renyi Entropy which generalizes the Hartley and Shannon entropies which is given by $R_{\delta}=\frac{1}{1-\delta} \log \left(\int_{0}^{1} f^{\delta}(x) d x\right)$, $\delta \neq 1$. The Renyi entropy in of the UGIW distribution is

$$
R_{\delta}=\frac{\delta}{1-\delta} \log \lambda+\frac{\delta-1}{1-\delta} \log \beta+\frac{\delta}{1-\delta} \log \left\{\sum_{i=0}^{2(\delta+1)}\left(\begin{array}{c}
2(\delta+1) \\
i
\end{array}\right) \frac{\alpha^{\delta-\frac{i}{\beta}-1} \lambda^{\delta-i-1}}{\delta^{\frac{i}{\beta}+1}} \Gamma\left(\frac{i}{\beta}+1\right)\right\} .
$$

The $\delta$-entropy, say $I_{\delta}(x)$, is defined by

$$
I_{\delta}(x)=\frac{1}{\delta-1} \log \left[1-\int_{0}^{1} f^{\delta}(x) d x\right], \quad \delta>0, \quad \delta \neq 1,
$$

and then it follows from equation (10).

The stress-strength reliability has been widely used in reliability analysis as the measure of the system performance under stress. In terms of probability, the stress-strength reliability can be obtained as

$$
R=P(Y<X),
$$

where $\mathrm{X}$ denotes strength of the system and Y denotes the stress applied on the system. The probability $\mathrm{R}$ can be used to compare the two random variables encountered in various applied disciplines. $\mathrm{R}$ for UGIW random variables $\left(X \sim \operatorname{UGIW}\left(\alpha_{1}, \lambda_{1}, \beta_{2}\right)\right.$ and $Y \sim$ $\left.\operatorname{UGIW}\left(\alpha_{2}, \lambda_{2}, \beta_{2}\right)\right)$ is given by

$$
R=\beta_{1} \sum_{i=0}^{\infty} \sum_{j=0}^{\infty}(-1)^{i+j} \frac{\alpha_{1}^{i+1} \alpha_{2}^{j} \lambda_{1}^{\beta_{1}(i+1)} \lambda_{2}^{\beta_{2} j}}{i ! j !} B e\left(2-\beta_{1}(i+1)-\beta_{2} j, \quad \beta_{1}(i+1)+\beta_{2} j\right),
$$

where $B(a, b)$, is the complete beta function and is defined by $B(a, b)=\int_{0}^{1} x^{a-1}(1-x)^{b-1} d x$. 


\subsection{Order statistics}

Let $X_{1}, X_{2}, \ldots, X_{n}$ be a random sample from UGIW distribution. Let $X_{1: n} \leq X_{2: n} \leq \ldots \leq$ $X_{n: n}$ be the order statistics from this random sample, then the $p d f f_{r: n}(x)$ of the $r^{\text {th }}$ order statistic, for $r=1,2, \ldots, n$, is obtained as follows:

$$
f_{r: n}(x)=\frac{n !}{(r-1) !(n-r) !} f(x)(F(x))^{r-1}(1-F(x))^{n-r},
$$

where $\mathrm{f}(\mathrm{x})$ and $\mathrm{F}(\mathrm{x})$ are the $P D F$ and $C D F$ of the UGIW distribution, respectively. The $\mathrm{PDF}$ of rth order statistic is

$$
\begin{aligned}
f_{r: n}(x) & =\frac{n !}{(r-1) !(n-r) !} \sum_{i=0}^{n-r}(-1)^{i}\left(\begin{array}{c}
n-r \\
i
\end{array}\right) f(x)(F(x))^{r+i-1} \\
& =\frac{\alpha \beta \lambda n !}{(r-1) !(n-r) !} \sum_{i=0}^{n-r}(-1)^{i}\left(\begin{array}{c}
n-r \\
i
\end{array}\right) \frac{1}{x^{2}}\left(\frac{\lambda(1-x)}{x}\right)^{\beta-1} \exp \left[-\alpha(r+i)\left(\frac{\lambda(1-x)}{x}\right)^{\beta}\right] .
\end{aligned}
$$

The $k t h$ moment of the $r t h$ order statistic is given by

$$
E\left(X_{r: n}^{k}\right)=\frac{n !}{(r-1) !(n-r) !} \sum_{i=0}^{n-r} \sum_{j=0}^{\infty}(-1)^{i+j}\left(\begin{array}{c}
n-r \\
i
\end{array}\right)\left(\begin{array}{c}
k+j-1 \\
j
\end{array}\right) \lambda^{-i} \alpha^{-\frac{i}{\beta}} \frac{\Gamma\left(\frac{i}{\beta}+1\right)}{(r+i)^{\frac{i}{\beta}+1}} .
$$

\section{Different methods of parameter estimation based on complete sample}

\subsection{Maximum likelihood estimation}

Here, the parameters of the UGIW distribution are estimated by using the method of maximum likelihood. Let $X_{1}, X_{2}, \ldots X_{n}$ be a random samples distributed according to the UGIW distribution, then the likelihood function can be written as

$$
L_{n}(\theta)=\prod_{i=1}^{n} f\left(x_{i}, \alpha, \lambda, \beta\right)
$$

By taking the natural logarithm, the log-likelihood function is obtained as;

$$
\log L_{n}(\theta)=n \ln (\alpha \lambda \beta)-2 \sum_{i=1}^{n} \ln \left(X_{i}\right)+(\beta-1) \sum_{i=1}^{n} \ln \left(\lambda u_{i}\right)-\alpha \sum_{i=1}^{n} \lambda^{\beta} u_{i}^{\beta}
$$

Let

$$
u_{i}=\frac{\left(1-x_{i}\right)}{x_{i}}
$$

Then the components of the score function are

$$
\begin{aligned}
& \frac{\partial L}{\partial \alpha}=\frac{n}{\alpha}-\sum_{i=1}^{n} \lambda^{\beta} u_{i}^{\beta}, \\
& \frac{\partial L}{\partial \lambda}=\frac{n}{\lambda}+(\beta-1) \sum_{i=1}^{n} \frac{u_{i}}{\lambda u_{i}}-\alpha \beta \sum_{i=1}^{n} \lambda^{\beta-1} u_{i}^{\beta}, \\
& \frac{\partial L}{\partial \beta}=\frac{n}{\beta}+\sum_{i=1}^{n} \ln \lambda u_{i}-\alpha \sum_{i=1}^{n}\left(\lambda u_{i}\right)^{\beta} \ln \lambda u_{i} .
\end{aligned}
$$


If we set these equations to zero and solve them simultaneously, we can compute the MLEs of the parameters $\alpha, \beta$ and $\lambda$. To solve these equations, it is usually more convenient to use nonlinear optimization methods such as quasi-Newton algorithm.

\subsection{Method of ordinary and weighted least squares}

The least square (LS) and the weighted least square (WLS) are well known methods used for estimating the unknown parameters (Swain, Venkatraman, and Wilson 1988). Here, we consider the two methods to estimate the unknown parameters of the $U G I W$ distribution. Let $x_{1}, x_{2} \ldots ., x_{n}$ be the ordered observations obtained from a sample of size $n$ from the $U G I W$ distribution., The LS and WLS estimates of $\alpha, \lambda$ and $\beta$ can be obtained by minimizing the following function with respect to $\alpha, \lambda$ and $\beta$, respectively

$$
S(\theta)=\sum_{i=1}^{n} \eta_{i}\left\{e^{-\alpha\left(\lambda u_{i}\right)^{\beta}}-\frac{i}{n+1}\right\}^{2}
$$

where $\theta=(\alpha, \beta, \lambda)$. The LS estimates denoted by $\hat{\alpha}_{L S E}, \hat{\lambda}_{L S E}$ and $\hat{\beta}_{L S E}$ and can be obtained by setting $\eta_{i}=1$, while we can obtain the $W L S$ estimates denoted by $\hat{\alpha}_{W L S} ; \hat{\lambda}_{W L S}$ and $\hat{\beta}_{W L S}$ by setting $\eta_{i}=\frac{(n+1)^{2}(n+2)}{i(n-i+1)}$. These estimates can also be obtained by solving the following equations

$$
\begin{aligned}
& \frac{\partial S(\theta)}{\partial \alpha}=\sum_{i=1}^{n} \eta_{i}\left\{e^{-\alpha\left(\lambda u_{i}\right)^{\beta}}-\frac{i}{n+1}\right\} \varphi_{1}\left(u_{i}, \theta\right)=0 \\
& \frac{\partial S(\theta)}{\partial \beta}=\sum_{i=1}^{n} \eta_{i}\left\{e^{-\alpha\left(\lambda u_{i}\right)^{\beta}}-\frac{i}{n+1}\right\} \varphi_{2}\left(u_{i}, \theta\right)=0 \\
& \frac{\partial S(\theta)}{\partial \lambda}=\sum_{i=1}^{n} \eta_{i}\left\{e^{-\alpha\left(\lambda u_{i}\right)^{\beta}}-\frac{i}{n+1}\right\} \varphi_{3}\left(u_{i}, \theta\right)=0
\end{aligned}
$$

where

$$
\begin{aligned}
& \varphi_{1}\left(u_{i}, \theta\right)=-\left(\lambda u_{i}\right)^{\beta} e^{-\alpha\left(\lambda u_{i}\right)^{\beta}} \\
& \varphi_{2}\left(u_{i}, \theta\right)=-\alpha\left(\lambda u_{i}\right)^{\beta} \ln \left(\lambda u_{i}\right) e^{-\alpha\left(\lambda u_{i}\right)^{\beta}} \\
& \varphi_{3}\left(u_{i}, \theta\right)=-\alpha \beta \lambda^{\beta-1} u_{i}^{\beta} e^{-\alpha\left(\lambda u_{i}\right)^{\beta}}
\end{aligned}
$$

where $u_{i}, i=1,2, \ldots . n$ are the order observations of $u_{i}$ as defined earlier.

\subsection{Method of percentile}

In this subsection, we estimate the unknown parameters of UGIW distribution by the percentile method. This method was first introduced by Kao (1958) for estimating Weibull parameters. Let $p_{i}=\frac{i}{n+1}$ be the estimate of $F\left(x_{i}, \theta\right)$, then the percentile estimates of the parameters of UGIW distribution are denoted by $\hat{\alpha}_{P E}, \hat{\lambda}_{P E}$ and $\hat{\beta}_{P E}$ and can be obtained by minimizing the following function

$$
P(\theta)=\sum_{i=1}^{n}\left\{x_{i}-\left[\frac{1}{\lambda}\left[-\frac{\ln \left(p_{i}\right)}{\alpha}\right]^{1 / \beta}+1\right]^{-1}\right\}^{2}
$$

with respect to $\alpha, \lambda$ and $\beta$ or equivalently by solving the following non-linear equations

$$
\frac{\partial P(\theta)}{\partial \alpha}=\sum_{i=1}^{n}\left\{x_{i}-\left[\frac{1}{\lambda} v_{i}^{1 / \beta}+1\right]^{-1}\right\} \varpi_{1}\left(v_{i}, \theta\right)=0
$$




$$
\begin{aligned}
& \frac{\partial P(\theta)}{\partial \beta}=\sum_{i=1}^{n}\left\{x_{i}-\left[\frac{1}{\lambda} v_{i}^{1 / \beta}+1\right]^{-1}\right\} \varpi_{2}\left(v_{i}, \theta\right)=0 \\
& \frac{\partial P(\theta)}{\partial \lambda}=\sum_{i=1}^{n}\left\{x_{i}-\left[\frac{1}{\lambda} v_{i}^{1 / \beta}+1\right]^{-1}\right\} \varpi_{3}\left(v_{i}, \theta\right)=0
\end{aligned}
$$

where $v_{i}=-\frac{\ln \left(p_{i}\right)}{\alpha}$

$$
\begin{aligned}
& \varpi_{1}\left(v_{i}, \theta\right)=\frac{v_{i}^{1 / \beta}}{\alpha \lambda \beta\left(\frac{1}{\lambda} v_{i}^{1 / \beta}+1\right)^{2}}, \\
& \varpi_{2}\left(v_{i}, \theta\right)=\frac{v_{i}^{1 / \beta} \ln v_{i}}{\lambda \beta^{2}\left(\frac{1}{\lambda} v_{i}^{1 / \beta}+1\right)^{2}}, \\
& \varpi_{3}\left(v_{i}, \theta\right)=\frac{v_{i}^{1 / \beta}}{\left(v_{i}^{1 / \beta}+\lambda\right)^{2}} .
\end{aligned}
$$

\subsection{Method of maximum product of spacing}

According to Cheng and Amin (1983), the maximum product of spacing (MPS) estimates of the unknown parameters of the $U G I W$ distribution can be obtained based on the idea of differences between the values of the cdf at consecutive data points. Based on a random sample of size $n$ from the UGIW distribution, the uniform spacings can be defined as follows

$$
D_{i}(\theta)=F\left(x_{i}, \theta\right)-F\left(x_{i-1}, \theta\right), \quad i=1,2, \ldots, n
$$

where $F(x, \theta)$ is the cdf given by $(2), F\left(x_{0}, \theta\right)=0$ and $F\left(x_{n+1}, \theta\right)=1$. The MPS estimates denoted by $\hat{\alpha}_{M P S}, \hat{\lambda}_{M P S}$ and $\hat{\beta}_{M P S}$ can be obtained by maximizing

$$
\begin{aligned}
M(\theta) & =\frac{1}{n+1} \sum_{i=1}^{n+1} \log D_{i}(\theta) \\
& =\frac{1}{n+1} \sum_{i=1}^{n+1} \log \left\{e^{-\alpha\left(\lambda u_{i}\right)^{\beta}}-e^{-\alpha\left(\lambda u_{i-1}\right)^{\beta}}\right\}
\end{aligned}
$$

with respect to $\alpha, \lambda$ and $\beta$ or by solving the following equations

$$
\begin{aligned}
& \frac{\partial M(\theta)}{\partial \alpha}=\frac{1}{n+1} \sum_{i=1}^{n+1} \frac{\varphi_{1}\left(u_{i}, \theta\right)-\varphi_{1}\left(u_{i-1}, \theta\right)}{D_{i}}=0 \\
& \frac{\partial M(\theta)}{\partial \beta}=\frac{1}{n+1} \sum_{i=1}^{n+1} \frac{\varphi_{2}\left(u_{i}, \theta\right)-\varphi_{2}\left(u_{i-1}, \theta\right)}{D_{i}}=0 \\
& \frac{\partial M(\theta)}{\partial \lambda}=\frac{1}{n+1} \sum_{i=1}^{n+1} \frac{\varphi_{3}\left(u_{i}, \theta\right)-\varphi_{3}\left(u_{i-1}, \theta\right)}{D_{i}}=0
\end{aligned}
$$

where $\varphi_{1}\left(u_{i}, \theta\right), \varphi_{2}\left(u_{i}, \theta\right)$ and $\varphi_{3}\left(u_{i}, \theta\right)$ are given by (13), (14) and (15).

\subsection{Method of Cramér-von-Mises}

The Cramér-von-Mises estimates (CMEs) denoted by $\hat{\alpha}_{C M E}, \hat{\lambda}_{C M E}$ and $\hat{\beta}_{C M E}$ of $\alpha, \lambda$ and $\beta$ can be obtained by minimizing the following function with respect to $\alpha, \lambda$ and $\beta$.

$$
C(\theta)=\frac{1}{12 n}+\sum_{i=1}^{n}\left\{e^{-\alpha\left(\lambda u_{i}\right)^{\beta}}-\frac{2 i-1}{2 n}\right\}^{2}
$$


These estimates also can be obtained by solving the following equations

$$
\begin{aligned}
& \frac{\partial C(\theta)}{\partial \alpha}=\sum_{i=1}^{n}\left\{e^{-\alpha\left(\lambda u_{i}\right)^{\beta}}-\frac{2 i-1}{2 n}\right\} \varphi_{1}\left(u_{i}, \theta\right)=0 \\
& \frac{\partial C(\theta)}{\partial \beta}=\sum_{i=1}^{n}\left\{e^{-\alpha\left(\lambda u_{i}\right)^{\beta}}-\frac{2 i-1}{2 n}\right\} \varphi_{2}\left(u_{i}, \theta\right)=0 \\
& \frac{\partial C(\theta)}{\partial \lambda}=\sum_{i=1}^{n}\left\{e^{-\alpha\left(\lambda u_{i}\right)^{\beta}}-\frac{2 i-1}{2 n}\right\} \varphi_{3}\left(u_{i}, \theta\right)=0
\end{aligned}
$$

where $\varphi_{1}\left(u_{i}, \theta\right), \varphi_{2}\left(u_{i}, \theta\right)$ and $\varphi_{3}\left(u_{i}, \theta\right)$ are defined by (13), (14) and (15).

\section{Simulation results for complete data}

It is not possible to compare the performance of the differernt estimators derived in the previous sections theoretically, therefore, we conduct a Monte Carlo simulation study to determine the best estimation method among six classical estimation methods. We generate 10,000 random samples of different sample sizes and different parameter values. We replicate the process 1000 times and obtain the average of the estimates and the MSE in each case. From Tables 2-7, it is noted that the maximum likelihood method of estimation performs better than other methods in terms of MSE in most of the cases. The next best performing estimator is the PE followed by LSE in most of the cases. Finally, we noted that the MSE decreases in all the methods of estimation as the sample size increases, which indicates that the all the methods of estimation are consistent. Form Tables 2-7, it is also observed that, PE gives the least AWs in case of $\alpha$ while for $\beta$ and $\lambda$, MLE performs better than other methods of estimation. It is also observed that MLE performs better than other methods of estimation in case of CPs in most of the cases.

Table 2: Average estimates, estimated average widths (AW) and coverage probabilities (CP) of the parameters based on ML estimates

\begin{tabular}{rlllllllll}
\multicolumn{1}{c}{$\mathrm{n}$} & $\alpha=1.5$ & AW & CP & $\lambda=2.5$ & AW & CP & $\beta=2$ & AW & CP \\
\hline 10 & $1.532(0.0155)$ & 0.0303 & 0.924 & $2.426(0.0135)$ & 0.0264 & 0.935 & $1.763(0.0126)$ & 0.0246 & 0.929 \\
20 & $1.530(0.0127)$ & 0.0248 & 0.926 & $2.453(0.0112)$ & 0.0219 & 0.937 & $1.823(0.0102)$ & 0.0199 & 0.932 \\
50 & $1.528(0.0106)$ & 0.0207 & 0.929 & $2.487(0.0098)$ & 0.0192 & 0.939 & $1.871(0.0086)$ & 0.0168 & 0.935 \\
150 & $1.517(0.0091)$ & 0.0178 & 0.931 & $2.494(0.0079)$ & 0.0154 & 0.942 & $1.901(0.0068)$ & 0.0133 & 0.937 \\
350 & $1.507(0.0075)$ & 0.0147 & 0.934 & $2.498(0.0043)$ & 0.0084 & 0.946 & $1.947(0.0049)$ & 0.0096 & 0.940 \\
500 & $1.503(0.0052)$ & 0.0101 & 0.937 & $2.499(0.0028)$ & 0.0054 & 0.948 & $1.992(0.0037)$ & 0.0072 & 0.942 \\
\hline $\mathrm{n}$ & $\alpha=2$ & AW & CP & $\lambda=3$ & AW & CP & $\beta=1.5$ & AW & CP \\
\hline 10 & $1.735(0.0196)$ & 0.0384 & 0.932 & $2.956(0.0126)$ & 0.0246 & 0.916 & $1.474(0.0103)$ & 0.0201 & 0.934 \\
20 & $1.764(0.0154)$ & 0.0301 & 0.937 & $2.959(0.0112)$ & 0.0219 & 0.918 & $1.476(0.0099)$ & 0.0194 & 0.935 \\
50 & $1.826(0.0120)$ & 0.0235 & 0.941 & $2.962(0.0092)$ & 0.0180 & 0.921 & $1.483(0.0089)$ & 0.0174 & 0.937 \\
150 & $1.874(0.0096)$ & 0.0188 & 0.943 & $2.969(0.0076)$ & 0.0148 & 0.924 & $1.486(0.0079)$ & 0.0154 & 0.938 \\
350 & $1.927(0.0078)$ & 0.0152 & 0.945 & $2.973(0.0053)$ & 0.0103 & 0.927 & $1.493(0.0048)$ & 0.0094 & 0.940 \\
500 & $1.993(0.0043)$ & 0.0084 & 0.947 & $2.998(0.0027)$ & 0.0052 & 0.929 & $1.499(0.0023)$ & 0.0045 & 0.943 \\
\hline $\mathrm{n}$ & $\alpha=0.9$ & AW & CP & $\lambda=2$ & AW & CP & $\beta=0.5$ & AW & CP \\
\hline 10 & $0.923(0.0152)$ & 0.0297 & 0.920 & $1.976(0.0072)$ & 0.0141 & 0.922 & $0.478(0.0088)$ & 0.0172 & 0.918 \\
20 & $0.919(0.0134)$ & 0.0262 & 0.926 & $1.981(0.0053)$ & 0.0103 & 0.924 & $0.486(0.0072)$ & 0.0141 & 0.922 \\
50 & $0.913(0.0112)$ & 0.0219 & 0.928 & $1.985(0.0046)$ & 0.0090 & 0.926 & $0.489(0.0068)$ & 0.0133 & 0.925 \\
150 & $0.908(0.0084)$ & 0.0164 & 0.934 & $1.989(0.0038)$ & 0.0074 & 0.927 & $0.492(0.0042)$ & 0.0082 & 0.927 \\
350 & $0.905(0.0038)$ & 0.0074 & 0.936 & $1.992(0.0026)$ & 0.0050 & 0.928 & $0.497(0.0031)$ & 0.0060 & 0.928 \\
500 & $0.903(0.0027)$ & 0.0052 & 0.939 & $1.998(0.0013)$ & 0.0025 & 0.930 & $0.501(0.0022)$ & 0.0043 & 0.930 \\
\hline
\end{tabular}


Table 3: Average estimates, estimated average widths (AW) and coverage probabilities (CP) of of the parameters based on WLS estimates

\begin{tabular}{rlllllllll}
\hline \multicolumn{1}{c}{$\mathrm{n}$} & $\alpha=1.5$ & $\mathrm{AW}$ & $\mathrm{CP}$ & $\lambda=2.5$ & $\mathrm{AW}$ & $\mathrm{CP}$ & $\beta=2$ & $\mathrm{AW}$ & $\mathrm{CP}$ \\
\hline 10 & $1.563(0.0266)$ & 0.0521 & 0.909 & $2.561(0.0241)$ & 0.0472 & 0.911 & $2.419(0.0246)$ & 0.0482 & 0.912 \\
20 & $1.559(0.0234)$ & 0.0458 & 0.912 & $2.557(0.0210)$ & 0.0411 & 0.913 & $2.391(0.0213)$ & 0.0417 & 0.915 \\
50 & $1.556(0.0204)$ & 0.0399 & 0.914 & $2.546(0.0189)$ & 0.0370 & 0.915 & $2.363(0.0172)$ & 0.0337 & 0.918 \\
150 & $1.548(0.0185)$ & 0.0362 & 0.916 & $2.531(0.0173)$ & 0.0339 & 0.919 & $2.289(0.0162)$ & 0.0317 & 0.921 \\
350 & $1.539(0.0152)$ & 0.0297 & 0.918 & $2.528(0.0143)$ & 0.0280 & 0.922 & $2.235(0.0137)$ & 0.0268 & 0.924 \\
500 & $1.531(0.0134)$ & 0.0262 & 0.921 & $2.522(0.0122)$ & 0.0239 & 0.926 & $2.164(0.0112)$ & 0.0219 & 0.928 \\
\hline $\mathrm{n}$ & alpha=2 & $\mathrm{AW}$ & $\mathrm{CP}$ & lambda=3 & $\mathrm{AW}$ & $\mathrm{CP}$ & $\beta=1.5$ & $\mathrm{AW}$ & $\mathrm{CP}$ \\
\hline 10 & $2.125(0.0259)$ & 0.0507 & 0.903 & $3.183(0.0263)$ & 0.0515 & 0.907 & $1.546(0.0271)$ & 0.0531 & 0.910 \\
20 & $2.121(0.0224)$ & 0.0439 & 0.905 & $3.178(0.0228)$ & 0.0446 & 0.910 & $1.537(0.0250)$ & 0.0490 & 0.913 \\
50 & $2.119(0.0196)$ & 0.0384 & 0.907 & $3.176(0.0202)$ & 0.0395 & 0.914 & $1.529(0.0233)$ & 0.0456 & 0.915 \\
150 & $2.116(0.0153)$ & 0.0299 & 0.910 & $3.168(0.0198)$ & 0.0388 & 0.917 & $1.521(0.0212)$ & 0.0415 & 0.919 \\
350 & $2.112(0.0134)$ & 0.0262 & 0.913 & $3.161(0.0172)$ & 0.0337 & 0.920 & $1.516(0.0192)$ & 0.0376 & 0.922 \\
500 & $2.109(0.0112)$ & 0.0219 & 0.918 & $3.159(0.0144)$ & 0.0282 & 0.923 & $1.513(0.0173)$ & 0.0339 & 0.925 \\
\hline $\mathrm{n}$ & $\alpha=0.9$ & $\mathrm{AW}$ & $\mathrm{CP}$ & $\lambda=2$ & $\mathrm{AW}$ & $\mathrm{CP}$ & $\beta=0.5$ & $\mathrm{AW}$ & $\mathrm{CP}$ \\
\hline 10 & $0.931(0.0210)$ & 0.0411 & 0.921 & $2.348(0.0161)$ & 0.0315 & 0.918 & $0.553(0.0176)$ & 0.0344 & 0.919 \\
20 & $0.928(0.0187)$ & 0.0366 & 0.925 & $2.339(0.0152)$ & 0.0297 & 0.921 & $0.547(0.0159)$ & 0.0311 & 0.920 \\
50 & $0.927(0.0158)$ & 0.0309 & 0.926 & $2.334(0.0137)$ & 0.0268 & 0.924 & $0.542(0.0131)$ & 0.0256 & 0.922 \\
150 & $0.919(0.0137)$ & 0.0268 & 0.929 & $2.326(0.0116)$ & 0.0227 & 0.926 & $0.536(0.0102)$ & 0.0199 & 0.924 \\
350 & $0.916(0.0106)$ & 0.0207 & 0.931 & $2.316(0.0093)$ & 0.0182 & 0.928 & $0.532(0.0099)$ & 0.0194 & 0.928 \\
500 & $0.913(0.0088)$ & 0.0172 & 0.934 & $2.310(0.0078)$ & 0.0152 & 0.932 & $0.528(0.0081)$ & 0.0158 & 0.932 \\
\hline
\end{tabular}

Table 4: Average estimates, estimated average widths (AW) and coverage probabilities (CP) of the parameters based on MPS estimates

\begin{tabular}{rlllllllll}
\hline \multicolumn{1}{c}{$\mathrm{n}$} & $\alpha=1.5$ & $\mathrm{AW}$ & $\mathrm{CP}$ & $\lambda=2.5$ & $\mathrm{AW}$ & $\mathrm{CP}$ & $\beta=2$ & $\mathrm{AW}$ & $\mathrm{CP}$ \\
\hline 10 & $1.557(0.0254)$ & 0.0497 & 0.915 & $2.548(0.0216)$ & 0.0423 & 0.920 & $2.351(0.0221)$ & 0.0433 & 0.923 \\
20 & $1.555(0.0212)$ & 0.0415 & 0.917 & $2.542(0.0198)$ & 0.0388 & 0.923 & $2.296(0.0167)$ & 0.0327 & 0.925 \\
50 & $1.547(0.0198)$ & 0.0388 & 0.920 & $2.535(0.0178)$ & 0.0348 & 0.925 & $2.241(0.0148)$ & 0.0290 & 0.927 \\
150 & $1.539(0.0176)$ & 0.0344 & 0.922 & $2.528(0.0154)$ & 0.0301 & 0.927 & $2.176(0.0134)$ & 0.0262 & 0.930 \\
350 & $1.532(0.0167)$ & 0.0327 & 0.923 & $2.519(0.0138)$ & 0.0270 & 0.930 & $2.125(0.0119)$ & 0.0233 & 0.932 \\
500 & $1.524(0.0146)$ & 0.0286 & 0.925 & $2.512(0.0129)$ & 0.0252 & 0.934 & $2.009(0.0098)$ & 0.0192 & 0.935 \\
\hline $\mathrm{n}$ & $\alpha=2$ & $\mathrm{AW}$ & $\mathrm{CP}$ & $\lambda=3$ & $\mathrm{AW}$ & $\mathrm{CP}$ & $\beta=1.5$ & $\mathrm{AW}$ & $\mathrm{CP}$ \\
\hline 10 & $2.113(0.0253)$ & 0.0495 & 0.927 & $2.794(0.0153)$ & 0.0299 & 0.936 & $1.524(0.0157)$ & 0.0307 & 0.932 \\
20 & $2.097(0.0233)$ & 0.0456 & 0.929 & $2.817(0.0146)$ & 0.0286 & 0.938 & $1.522(0.0136)$ & 0.0266 & 0.935 \\
50 & $2.056(0.0173)$ & 0.0339 & 0.931 & $2.876(0.0113)$ & 0.0221 & 0.941 & $1.519(0.0114)$ & 0.0223 & 0.938 \\
150 & $2.019(0.0164)$ & 0.0321 & 0.933 & $2.934(0.0099)$ & 0.0194 & 0.943 & $1.517(0.0096)$ & 0.0188 & 0.942 \\
350 & $2.013(0.0122)$ & 0.0239 & 0.935 & $2.996(0.0074)$ & 0.0145 & 0.946 & $1.512(0.0089)$ & 0.0174 & 0.944 \\
500 & $2.009(0.0089)$ & 0.0174 & 0.938 & $3.005(0.0052)$ & 0.0101 & 0.947 & $1.507(0.0051)$ & 0.0099 & 0.946 \\
\hline $\mathrm{n}$ & $\alpha=0.9$ & $\mathrm{AW}$ & $\mathrm{CP}$ & $\lambda=2$ & $\mathrm{AW}$ & $\mathrm{CP}$ & $\beta=0.5$ & $\mathrm{AW}$ & $\mathrm{CP}$ \\
\hline 10 & $0.858(0.0203)$ & 0.0397 & 0.929 & $1.946(0.0149)$ & 0.0292 & 0.935 & $0.526(0.0162)$ & 0.0317 & 0.933 \\
20 & $0.862(0.0176)$ & 0.0344 & 0.931 & $1.949(0.0136)$ & 0.0266 & 0.936 & $0.519(0.0148)$ & 0.0290 & 0.936 \\
50 & $0.865(0.0148)$ & 0.0290 & 0.933 & $1.958(0.0125)$ & 0.0245 & 0.938 & $0.515(0.0123)$ & 0.0241 & 0.937 \\
150 & $0.871(0.0127)$ & 0.0248 & 0.935 & $1.968(0.0096)$ & 0.0188 & 0.940 & $0.511(0.0109)$ & 0.0213 & 0.941 \\
350 & $0.878(0.0098)$ & 0.0192 & 0.937 & $1.973(0.0076)$ & 0.0148 & 0.942 & $0.507(0.0095)$ & 0.0186 & 0.943 \\
500 & $0.884(0.0078)$ & 0.0152 & 0.939 & $1.987(0.0053)$ & 0.0103 & 0.943 & $0.505(0.0072)$ & 0.0141 & 0.945 \\
\hline
\end{tabular}


Table 5: Average estimates, estimated average widths (AW) and coverage probabilities (CP) of the parameters based on CM estimates

\begin{tabular}{rlllllllll}
\hline \multicolumn{1}{r}{} & $\alpha=1.5$ & AW & CP & $\lambda=2.5$ & AW & CP & $\beta=2$ & AW & CP \\
\hline 10 & $1.584(0.0223)$ & 0.0437 & 0.910 & $2.569(0.0186)$ & 0.0364 & 0.916 & $1.563(0.0189)$ & 0.0370 & 0.917 \\
20 & $1.580(0.0192)$ & 0.0376 & 0.913 & $2.563(0.0170)$ & 0.0333 & 0.918 & $1.593(0.0157)$ & 0.0307 & 0.919 \\
50 & $1.574(0.0174)$ & 0.0341 & 0.915 & $2.557(0.0159)$ & 0.0311 & 0.919 & $1.646(0.0139)$ & 0.0272 & 0.923 \\
150 & $1.569(0.0153)$ & 0.0299 & 0.917 & $2.549(0.0142)$ & 0.0278 & 0.923 & $1.694(0.0124)$ & 0.0243 & 0.927 \\
350 & $1.553(0.0124)$ & 0.0243 & 0.919 & $2.543(0.0126)$ & 0.0246 & 0.926 & $1.724(0.0101)$ & 0.0197 & 0.932 \\
500 & $1.547(0.0104)$ & 0.0203 & 0.923 & $2.537(0.0099)$ & 0.0194 & 0.934 & $1.791(0.0086)$ & 0.0168 & 0.936 \\
\hline $\mathrm{n}$ & $\alpha=2$ & AW & CP & $\lambda=3$ & AW & CP & $\beta=1.5$ & AW & CP \\
\hline 10 & $2.241(0.0283)$ & 0.0554 & 0.904 & $3.246(0.0302)$ & 0.0591 & 0.903 & $1.563(0.0298)$ & 0.0584 & 0.901 \\
20 & $2.221(0.0263)$ & 0.0515 & 0.906 & $3.239(0.0248)$ & 0.0486 & 0.906 & $1.559(0.0289)$ & 0.0566 & 0.904 \\
50 & $2.194(0.0231)$ & 0.0452 & 0.909 & $3.229(0.0232)$ & 0.0454 & 0.908 & $1.551(0.0276)$ & 0.0540 & 0.907 \\
150 & $2.138(0.0201)$ & 0.0393 & 0.910 & $3.221(0.0220)$ & 0.0431 & 0.910 & $1.543(0.0243)$ & 0.0476 & 0.909 \\
350 & $2.132(0.0162)$ & 0.0317 & 0.913 & $3.216(0.0199)$ & 0.0390 & 0.913 & $1.536(0.0225)$ & 0.0441 & 0.911 \\
500 & $2.126(0.0134)$ & 0.0262 & 0.918 & $3.212(0.0189)$ & 0.0370 & 0.918 & $1.532(0.0204)$ & 0.0399 & 0.915 \\
\hline $\mathrm{n}$ & $\alpha=0.9$ & AW & $\mathrm{CP}$ & $\lambda=2$ & $\mathrm{AW}$ & $\mathrm{CP}$ & $\beta=0.5$ & AW & $\mathrm{CP}$ \\
\hline 10 & $0.967(0.0233)$ & 0.0456 & 0.912 & $1.812(0.0178)$ & 0.0348 & 0.920 & $0.576(0.0186)$ & 0.0364 & 0.916 \\
20 & $0.961(0.0192)$ & 0.0376 & 0.916 & $1.815(0.0167)$ & 0.0327 & 0.926 & $0.569(0.0163)$ & 0.0319 & 0.918 \\
50 & $0.958(0.0165)$ & 0.0323 & 0.919 & $1.819(0.0149)$ & 0.0292 & 0.931 & $0.564(0.0146)$ & 0.0286 & 0.922 \\
150 & $0.952(0.0146)$ & 0.0286 & 0.921 & $1.824(0.0128)$ & 0.0250 & 0.935 & $0.559(0.0138)$ & 0.0270 & 0.925 \\
350 & $0.948(0.0114)$ & 0.0223 & 0.925 & $1.829(0.0099)$ & 0.0194 & 0.938 & $0.554(0.0112)$ & 0.0219 & 0.929 \\
500 & $0.946(0.0092)$ & 0.0180 & 0.929 & $1.834(0.0083)$ & 0.0162 & 0.941 & $0.547(0.0096)$ & 0.0188 & 0.931 \\
\hline
\end{tabular}

Table 6: Average estimates, estimated average widths (AW) and coverage probabilities (CP) of the parameters based on PE estimates

\begin{tabular}{rlllllllll}
\hline \multicolumn{1}{r|}{$\mathrm{n}$} & $\alpha=1.5$ & $\mathrm{AW}$ & $\mathrm{CP}$ & $\lambda=2.5$ & $\mathrm{AW}$ & $\mathrm{CP}$ & $\beta=2$ & AW & $\mathrm{CP}$ \\
\hline 10 & $1.549(0.0153)$ & 0.0299 & 0.932 & $2.403(0.0156)$ & 0.0305 & 0.922 & $1.759(0.0156)$ & 0.0305 & 0.922 \\
20 & $1.541(0.0134)$ & 0.0262 & 0.933 & $2.458(0.0130)$ & 0.0254 & 0.926 & $1.826(0.0126)$ & 0.0246 & 0.925 \\
50 & $1.533(0.0112)$ & 0.0219 & 0.934 & $2.478(0.0104)$ & 0.0203 & 0.928 & $1.869(0.0094)$ & 0.0184 & 0.927 \\
150 & $1.521(0.0094)$ & 0.0184 & 0.937 & $2.482(0.0082)$ & 0.0160 & 0.932 & $1.923(0.0071)$ & 0.0139 & 0.931 \\
350 & $1.516(0.0082)$ & 0.0160 & 0.940 & $2.496(0.0058)$ & 0.0113 & 0.935 & $1.968(0.0062)$ & 0.0121 & 0.934 \\
500 & $1.508(0.0064)$ & 0.0125 & 0.942 & $2.501(0.0042)$ & 0.0082 & 0.938 & $2.003(0.0048)$ & 0.0094 & 0.938 \\
\hline $\mathrm{n}$ & $\alpha=2$ & $\mathrm{AW}$ & $\mathrm{CP}$ & $\lambda=3$ & $\mathrm{AW}$ & $\mathrm{CP}$ & $\beta=1.5$ & $\mathrm{AW}$ & $\mathrm{CP}$ \\
\hline 10 & $1.694(0.0201)$ & 0.0393 & 0.912 & $2.693(0.0143)$ & 0.0280 & 0.923 & $1.478(0.0124)$ & 0.0243 & 0.927 \\
20 & $1.737(0.0189)$ & 0.0370 & 0.914 & $2.721(0.0126)$ & 0.0246 & 0.927 & $1.483(0.0106)$ & 0.0207 & 0.931 \\
50 & $1.796(0.0153)$ & 0.0299 & 0.916 & $2.796(0.0099)$ & 0.0194 & 0.930 & $1.486(0.0093)$ & 0.0182 & 0.935 \\
150 & $1.834(0.0121)$ & 0.0273 & 0.919 & $2.816(0.0086)$ & 0.0168 & 0.932 & $1.493(0.0087)$ & 0.0170 & 0.936 \\
350 & $1.896(0.0097)$ & 0.0190 & 0.923 & $2.873(0.0063)$ & 0.0123 & 0.936 & $1.599(0.0076)$ & 0.0148 & 0.941 \\
500 & $1.926(0.0076)$ & 0.0148 & 0.928 & $2.934(0.0041)$ & 0.0080 & 0.940 & $1.503(0.0061)$ & 0.0119 & 0.944 \\
\hline $\mathrm{n}$ & $\alpha=0.9$ & $\mathrm{AW}$ & $\mathrm{CP}$ & $\lambda=2$ & $\mathrm{AW}$ & $\mathrm{CP}$ & $\beta=0.5$ & $\mathrm{AW}$ & $\mathrm{CP}$ \\
\hline 10 & $0.931(0.0198)$ & 0.0388 & 0.909 & $1.956(0.0123)$ & 0.0241 & 0.930 & $0.463(0.0103)$ & 0.0201 & 0.934 \\
20 & $0.928(0.0162)$ & 0.0317 & 0.911 & $1.963(0.0113)$ & 0.0221 & 0.934 & $0.476(0.0092)$ & 0.0180 & 0.936 \\
50 & $0.926(0.0126)$ & 0.0246 & 0.916 & $1.979(0.0092)$ & 0.0180 & 0.938 & $0.482(0.0079)$ & 0.0154 & 0.942 \\
150 & $0.917(0.0097)$ & 0.0190 & 0.919 & $1.982(0.0078)$ & 0.0152 & 0.941 & $0.487(0.0068)$ & 0.0133 & 0.943 \\
350 & $0.909(0.0073)$ & 0.0143 & 0.922 & $1.993(0.0061)$ & 0.0119 & 0.943 & $0.492(0.0045)$ & 0.0088 & 0.946 \\
500 & $0.905(0.0043)$ & 0.0084 & 0.925 & $2.005(0.0033)$ & 0.0064 & 0.945 & $0.498(0.0032)$ & 0.0062 & 0.947 \\
\hline
\end{tabular}


Table 7: Average estimates,estimated average widths (AW) and coverage probabilities (CP) of the parameters based on LS estimates

\begin{tabular}{rlllllllll}
\hline $\mathrm{n}$ & $\alpha=1.5$ & $\mathrm{AW}$ & $\mathrm{CP}$ & $\lambda=2.5$ & $\mathrm{AW}$ & $\mathrm{CP}$ & $\beta=2$ & $\mathrm{AW}$ & $\mathrm{CP}$ \\
\hline 10 & $1.543(0.0186)$ & 0.0364 & 0.916 & $2.523(0.0153)$ & 0.0299 & 0.926 & $2.213(0.0173)$ & 0.0339 & 0.918 \\
20 & $1.538(0.0173)$ & 0.0339 & 0.918 & $2.519(0.0132)$ & 0.0258 & 0.930 & $2.198(0.0154)$ & 0.0301 & 0.921 \\
50 & $1.534(0.0141)$ & 0.0276 & 0.923 & $2.517(0.0099)$ & 0.0194 & 0.934 & $2.136(0.0132)$ & 0.0258 & 0.925 \\
150 & $1.529(0.0126)$ & 0.0246 & 0.925 & $2.515(0.0076)$ & 0.0148 & 0.937 & $2.107(0.0102)$ & 0.0199 & 0.928 \\
350 & $1.513(0.0097)$ & 0.0190 & 0.929 & $2.512(0.0051)$ & 0.0099 & 0.939 & $2.053(0.0089)$ & 0.0174 & 0.932 \\
500 & $1.506(0.0078)$ & 0.0152 & 0.932 & $2.503(0.0044)$ & 0.0086 & 0.942 & $2.007(0.0076)$ & 0.0148 & 0.933 \\
\hline $\mathrm{n}$ & $\alpha=2$ & $\mathrm{AW}$ & $\mathrm{CP}$ & $\lambda=3$ & $\mathrm{AW}$ & $\mathrm{CP}$ & $\beta=1.5$ & $\mathrm{AW}$ & $\mathrm{CP}$ \\
\hline 10 & $2.124(0.0213)$ & 0.0417 & 0.916 & $3.172(0.0213)$ & 0.0417 & 0.916 & $1.536(0.0163)$ & 0.0319 & 0.923 \\
20 & $2.093(0.0178)$ & 0.0348 & 0.918 & $3.153(0.0112)$ & 0.0219 & 0.918 & $1.531(0.0121)$ & 0.0237 & 0.927 \\
50 & $2.074(0.0153)$ & 0.0299 & 0.923 & $3.124(0.0092)$ & 0.0180 & 0.921 & $1.529(0.0092)$ & 0.0180 & 0.930 \\
150 & $2.067(0.0123)$ & 0.0241 & 0.925 & $3.096(0.0086)$ & 0.0168 & 0.923 & $1.526(0.0078)$ & 0.0152 & 0.932 \\
350 & $2.036(0.0099)$ & 0.0194 & 0.928 & $3.017(0.0078)$ & 0.0152 & 0.925 & $1.512(0.0069)$ & 0.0135 & 0.936 \\
500 & $2.007(0.0072)$ & 0.0141 & 0.931 & $3.006(0.0066)$ & 0.0129 & 0.928 & $1.509(0.0049)$ & 0.0135 & 0.940 \\
\hline $\mathrm{n}$ & $\alpha=0.9$ & $\mathrm{AW}$ & $\mathrm{CP}$ & $\lambda=2$ & $\mathrm{AW}$ & $\mathrm{CP}$ & $\beta=0.5$ & $\mathrm{AW}$ & $\mathrm{CP}$ \\
\hline 10 & $0.931(0.0136)$ & 0.0266 & 0.934 & $2.167(0.0099)$ & 0.0194 & 0.939 & $0.537(0.0116)$ & 0.0227 & 0.931 \\
20 & $0.927(0.0112)$ & 0.0219 & 0.937 & $2.136(0.0082)$ & 0.0160 & 0.941 & $0.526(0.0096)$ & 0.0188 & 0.933 \\
50 & $0.921(0.0089)$ & 0.0174 & 0.940 & $2.111(0.0069)$ & 0.0135 & 0.943 & $0.521(0.0081)$ & 0.0158 & 0.936 \\
150 & $0.918(0.0069)$ & 0.0135 & 0.942 & $2.093(0.0047)$ & 0.0092 & 0.945 & $0.519(0.0056)$ & 0.0109 & 0.939 \\
350 & $0.910(0.0058)$ & 0.0113 & 0.945 & $2.024(0.0038)$ & 0.0074 & 0.946 & $0.516(0.0048)$ & 0.0094 & 0.942 \\
500 & $0.901(0.0042)$ & 0.0082 & 0.947 & $2.003(0.0026)$ & 0.0050 & 0.948 & $0.501(0.0033)$ & 0.0064 & 0.943 \\
\hline
\end{tabular}

\section{Application with complete data}

In this section, we provide one application to real data set to illustrate the importance of the UGIW distribution presented in Section 2. The MLEs of the model parameters are computed and goodness-of-fit statistics with rival models are compared.

The data set consists of 63 observations of the strengths of $1.5 \mathrm{~cm}$ glass fibers taken from Smith and Naylor (1987). The data are: $0.55,0.74,0.77,0.81,0.84,0.93,1.04,1.11,1.13$, $1.24,1.25,1.27,1.28,1.29,1.30,1.36,1.39,1.42,1.48,1.48,1.49,1.49,1.49,1.50,1.50,1.50$, $1.51,1.52,1.53,1.54,1.55,1.55,1.58,1.59,1.60,1.61,1.61,1.61,1.61,1.62,1.62,1.63,1.64$, $1.66,1.66,1.67,1.68,1.68,1.69,1.70,1.70,1.73,1.76,1.76,1.77,1.78,1.81,1.82,1.84,1.84$, $1.89,2.00,2.01,2.24$.

We divide the data by 2.24 , to get the data set between 0 and 1 .

We compare the fits of the $U G I W$ distribution with some comprting models and their densities are given by:

- The unit-Birnbaum-Saunders distribution (UB):

$$
f(x)=\frac{1}{2 x \alpha \sqrt{2 \pi}}\left[\left(\frac{-\beta}{\log x}\right)^{1 / 2}+\left(\frac{-\beta}{\log x}\right)^{3 / 2}\right] \exp \left\{\frac{1}{2 \alpha^{2}}\left(\frac{\log x}{\beta}+\frac{\beta}{\log x}+2\right)\right\}
$$

- The unit-Weibull distribution (UW):

$$
f(x)=\frac{\alpha \beta}{x}(-\log x)^{\beta-1} \exp \left\{-\alpha(-\log x)^{\beta}\right\}
$$

- Unit-Gompertz distribution (UG):

$$
f(x)=\alpha \beta x^{-(\beta+1)} \exp \left\{-\alpha\left(x^{-\beta}-1\right)\right\}
$$

- Unit Burr-III distribution:

$$
f(x)=\frac{\lambda b}{x^{2}}\left[1+\left(\frac{1}{x}-1\right)^{b}\right]^{-\lambda-1}\left(\frac{1}{x}-1\right)^{b-1}
$$


- Kumaraswamy Distribution:

$$
f(x)=\alpha \beta x^{\beta-1}\left(1-x^{\beta}\right)^{\alpha-1}
$$

The fitted models are compared using goodness-of-fit measures, namely: the maximized log-likelihood under the model $(-\hat{l})$, Cramèr-Von Mises $(C V M)$, Anderson-Darling $(A D)$, Kolmogorov-Smirnov $(K S)$ statistic and its p-value $(P V)$. It is clear that the $U G I W$ distribution fits very well the strengths of glass fibers data.

Table 8: Goodness-of-fit statistics for strengths of glass fibers dataset

\begin{tabular}{llllll}
\hline Model & $-\hat{l}$ & CVM & AD & KS & PV \\
\hline NGIWD & 176.130 & 0.043 & 0.1447 & 0.1028 & 0.9949 \\
UBS & 177.902 & 0.049 & 0.1733 & 0.1268 & 0.9522 \\
UG & 176.334 & 0.041 & 0.1526 & 0.1093 & 0.9312 \\
UW & 176.986 & 0.048 & 0.1577 & 0.1124 & 0.9383 \\
KW & 177.261 & 0.045 & 0.1622 & 0.1179 & 0.9426 \\
Unit-BurII & 177.862 & 0.048 & 0.1668 & 0.1213 & 0.9498 \\
\hline
\end{tabular}

Next, we obtain the estimates of the unknown parameters of the $U G I W$ distribution using six methods of estimation and the values of $-\hat{l}, \mathrm{KS}$ and the corresponding PV are displayed in Table 9 for strengths of glass fibers data set. The values in Table 9 reveal that the MLE method can be used to estimate the parameters of the UGIW distribution. However, all estimation methods perform well.

Table 9: The parameters estimate of the UGIW model using different methods of estimation and $\hat{l}, \mathrm{KS}$ statistic and corresponding PV

\begin{tabular}{lllllll}
\hline Method & $\alpha$ & $\lambda$ & $\beta$ & $-\hat{l}$ & KS & PV \\
\hline MLE & 1.4683 & 0.2373 & 0.8634 & 176.130 & 0.1028 & 0.9949 \\
WLSE & 1.3641 & 0.1673 & 0.5343 & 176.153 & 0.1047 & 0.9923 \\
PCS & 1.5718 & 0.3674 & 0.9341 & 176.096 & 0.1067 & 0.9836 \\
MPSE & 2.3641 & 0.5346 & 1.2467 & 176.273 & 0.1086 & 0.9712 \\
CMES & 0.9769 & 0.4367 & 1.1364 & 176.530 & 0.1121 & 0.9865 \\
LSEs & 2.5263 & 0.9652 & 2.0366 & 177.956 & 0.1203 & 0.9124 \\
\hline
\end{tabular}

\section{Maximum likelihood estimation with right censored data}

Let us consider $X=\left(X_{1}, X_{2}, \ldots, X_{n}\right)^{T}$ a sample from $U G I W$ distribution with parameter vector $\theta=(\alpha, \lambda, \beta)^{T}$ which can contain right censored data with fixed censoring time $\tau$. Each $X_{i}$ can be written as $X_{i}=\left(x_{i}, \Delta_{i}\right)$ where

$$
\Delta_{i}= \begin{cases}0 & \text { if } x_{i} \text { is a censoring time } \\ 1 & \text { if } x_{i} \text { is a failure time }\end{cases}
$$

The right censoring is assumed to be non informative, so the log-likelihood function can be written as:

$$
\begin{aligned}
L_{n}(\theta) & =\sum_{i=1}^{n} \Delta_{i} \ln h\left(x_{i}, \theta\right)+\sum_{i=1}^{n} \ln S\left(x_{i}, \theta\right) \\
& =\sum_{i=1}^{n} \Delta_{i}\left[\begin{array}{c}
\ln (\alpha \lambda \beta)-2 \ln \left(u_{i}\right)+(\beta-1) \ln (\lambda) \\
-\alpha\left(\lambda u_{i}\right)^{\beta}-\ln \left(1-\exp \left\{-\alpha\left(\lambda u_{i}\right)^{\beta}\right\}\right)
\end{array}\right]+\sum_{i=1}^{n} \ln \left(1-\exp \left\{-\alpha\left(\lambda u_{i}\right)^{\beta}\right\}\right)
\end{aligned}
$$


The maximum likelihood estimators $\widehat{\alpha}, \hat{\lambda}$ and $\widehat{\beta}$ of the unknown parameters $\alpha, \lambda$ and $\beta$ can be derived from the nonlinear following score equations:

$$
\begin{aligned}
& \frac{\partial L}{\partial \alpha}=\sum_{i=1}^{n} \Delta_{i}\left[\frac{1}{\alpha}-\frac{\left(\lambda u_{i}\right)^{\beta}}{1-e^{-\alpha\left(\lambda u_{i}\right)^{\beta}}}\right]+\sum_{i=1}^{n} \frac{\left(\lambda u_{i}\right)^{\beta} e^{-\alpha\left(\lambda u_{i}\right)^{\beta}}}{1-e^{-\alpha\left(\lambda u_{i}\right)^{\beta}}}=0 \\
& \frac{\partial L}{\partial \lambda}=\sum_{i=1}^{n} \Delta_{i}\left[\frac{\beta}{\lambda}-\frac{\alpha \beta \lambda^{\beta-1} u_{i}^{\beta}}{1-e^{-\alpha\left(\lambda u_{i}\right)^{\beta}}}\right]+\alpha \beta \lambda^{\beta-1} \sum_{i=1}^{n} \frac{u_{i}^{\beta} e^{-\alpha\left(\lambda u_{i}\right)^{\beta}}}{1-e^{-\alpha\left(\lambda u_{i}\right)^{\beta}}}=0 \\
& \frac{\partial L}{\partial \beta}=\sum_{i=1}^{n} \Delta_{i}\left[\frac{1}{\beta}+\ln \lambda u_{i}-\frac{\alpha\left(\lambda u_{i}\right)^{\beta} \ln \left(\lambda u_{i}\right)}{1-e^{-\alpha\left(\lambda u_{i}\right)^{\beta}}}\right]+\alpha \sum_{i=1}^{n} \frac{\left(\lambda u_{i}\right)^{\beta} \ln \left(\lambda u_{i}\right) e^{-\alpha\left(\lambda u_{i}\right)^{\beta}}}{1-e^{-\alpha\left(\lambda u_{i}\right)^{\beta}}}=0
\end{aligned}
$$

The explicit form of $\widehat{\alpha}, \hat{\lambda}$ and $\widehat{\beta}$ cannot be obtained, so we use numerical methods.

\section{Estimated Fisher information matrix}

The components of the estimated information matrix $I=\left(\hat{\imath}_{i j}\right)_{(3 \times 3)}$ are obtained by

$$
\begin{aligned}
& \hat{\imath}_{11}=\frac{1}{n} \sum_{i=1}^{n} \Delta_{i}\left(\frac{1}{\alpha}-\frac{\left(\lambda u_{i}\right)^{\beta}}{1-e^{-\alpha\left(\lambda u_{i}\right)^{\beta}}}\right)^{2} \\
& \hat{\imath}_{22}=\frac{1}{n} \sum_{i=1}^{n} \Delta_{i}\left(\frac{\beta}{\lambda}-\frac{\alpha \beta \lambda^{\beta-1} u_{i}^{\beta}}{1-e^{-\alpha\left(\lambda u_{i}\right)^{\beta}}}\right)^{2} \\
& \hat{\imath}_{33}=\frac{1}{n} \sum_{i=1}^{n} \Delta_{i}\left(\frac{1}{\beta}+\ln \lambda u_{i}-\frac{\alpha\left(\lambda u_{i}\right)^{\beta} \ln \left(\lambda u_{i}\right)}{1-e^{-\alpha\left(\lambda u_{i}\right)^{\beta}}}\right)^{2} \\
& \hat{\imath}_{12}=\hat{\imath}_{21}=\frac{1}{n} \sum_{i=1}^{n} \Delta_{i}\left(\frac{1}{\alpha}-\frac{\left(\lambda u_{i}\right)^{\beta}}{1-e^{-\alpha\left(\lambda u_{i}\right)^{\beta}}}\right)\left(\frac{\beta}{\lambda}-\frac{\alpha \beta \lambda^{\beta-1} u_{i}^{\beta}}{1-e^{-\alpha\left(\lambda u_{i}\right)^{\beta}}}\right) \\
& \hat{\imath}_{13}=\hat{\imath}_{31}=\frac{1}{n} \sum_{i=1}^{n} \Delta_{i}\left(\frac{1}{\alpha}-\frac{\left(\lambda u_{i}\right)^{\beta}}{1-e^{-\alpha\left(\lambda u_{i}\right)^{\beta}}}\right)\left(\frac{1}{\beta}+\ln \lambda u_{i}-\frac{\alpha\left(\lambda u_{i}\right)^{\beta} \ln \left(\lambda u_{i}\right)}{1-e^{-\alpha\left(\lambda u_{i}\right)^{\beta}}}\right) \\
& \hat{\imath}_{23}=\hat{\imath}_{32}=\frac{1}{n} \sum_{i=1}^{n} \Delta_{i}\left(\frac{\beta}{\lambda}-\frac{\alpha \beta \lambda^{\beta-1} u_{i}^{\beta}}{1-e^{-\alpha\left(\lambda u_{i}\right)^{\beta}}}\right)\left(\frac{1}{\beta}+\ln \lambda u_{i}-\frac{\alpha\left(\lambda u_{i}\right)^{\beta} \ln \left(\lambda u_{i}\right)}{1-e^{-\alpha\left(\lambda u_{i}\right)^{\beta}}}\right)
\end{aligned}
$$

where $\alpha, \lambda$ and $\beta$ are replaced by their MLEs $\widehat{\alpha}, \widehat{\lambda}$ and $\hat{\beta}$.

\section{Test statistic for right censored data}

Let $X_{1}, \ldots, X_{n}$ be $n$ i.i.d. random variables grouped into $k$ classes $I_{j}$. To assess the adequacy of a parametric model $F_{0}$, we consider

$H_{0}: \quad P\left(X_{i} \leq x \mid H_{0}\right)=F_{0}(x ; \theta), x \geq 0, \quad \theta=\left(\theta_{1}, \ldots, \theta_{s}\right)^{T} \in \Theta \subset R^{s}$

when data are right censored and the parameter vector $\theta$ is unknown, Bagdonavičius and Nikulin (2011) proposed a statistic test $Y^{2}$ based on the vector

$$
Z_{j}=\frac{1}{\sqrt{n}}\left(U_{j}-e_{j}\right), j=1,2, \ldots, k, \text { with } k \succ s .
$$


This represents the differences between the observed and the expected numbers of failures $\left(U_{j}\right.$ and $\left.e_{j}\right)$ to fall into these grouping intervals $I_{j}=\left(a_{j-1}, a_{j}\right]$ with $a_{0}=0, a_{k}=\tau$, where $\tau$ is a finite time. The authors considered $a_{j}$ as random data functions such as the $k$ intervals chosen have equal expected numbers of failures $e_{j}$.

The statistic test $Y^{2}$ is defined by

$$
Y^{2}=Z^{T} \widehat{\Sigma}^{-} Z=\sum_{j=1}^{k} \frac{\left(U_{j}-e_{j}\right)^{2}}{U_{j}}+Q
$$

where $Z=\left(Z_{1}, \ldots, Z_{k}\right)^{T}$ and $\widehat{\Sigma}^{-}$is a generalized inverse of the covariance matrix $\widehat{\Sigma}$ and

$$
\begin{aligned}
Q & =W^{T} \widehat{G}^{-} W \quad \widehat{A}_{j}=U_{j} / n, \quad U_{j}=\sum_{i: X_{i} \in I_{j}} \Delta_{i}, \\
W & =\left(W_{1}, \ldots, W_{s}\right)^{T}, \quad \widehat{G}=\left[\widehat{g}_{l l^{\prime}}\right]_{s x s}, \quad \widehat{g}_{l l^{\prime}}=\widehat{i}_{l l^{\prime}}-\sum_{j=1}^{k} \widehat{C}_{l j} \widehat{C}_{l^{\prime} j} \hat{A}_{j}^{-1}, \\
\widehat{C}_{l j} & =\frac{1}{n} \sum_{i: X_{i} \in I_{j}} \Delta_{i} \frac{\partial}{\partial \theta} \ln h\left(u_{i}, \widehat{\theta}\right), \quad \widehat{i}_{l l^{\prime}}=\frac{1}{n} \sum_{i=1}^{n} \Delta_{i} \frac{\partial \ln h\left(u_{i}, \widehat{\theta}\right)}{\partial \theta_{l}} \frac{\partial \ln h\left(u_{i}, \widehat{\theta}\right)}{\partial \theta_{l^{\prime}}}, \\
\widehat{W}_{l} & =\sum_{j=1}^{k} \widehat{C}_{l j} \widehat{A}_{j}^{-1} Z_{j}, \quad l, l^{\prime}=1, \ldots, s
\end{aligned}
$$

$\widehat{\theta}$ is the maximum likelihood estimator of $\theta$ on initial non-grouped data.

Under the null hypothesis $H_{0}$, the limit distribution of the statistic $Y^{2}$ is chi-square with $k=\operatorname{rank}(\Sigma)$ degrees of freedom. The description and applications of modified chi-square tests are discussed in Voinov, Nikulin, and Balakrishnan (2013).

The interval limits $a_{j}$ for grouping data into $j$ classes $I_{j}$ are considered as data functions and defined by

$$
\hat{a}_{j}=H^{-1}\left(\frac{E_{j}-\sum_{l=1}^{i-1} H\left(u_{l}, \theta\right)}{n-i+1}, \hat{\theta}\right), \quad \hat{a}_{k}=\max \left(X_{(n), \tau}\right)
$$

such that the expected failure times $e_{j}$ to fall into these intervals are $e_{j}=\frac{E_{k}}{k}$ for any $j$ with $E_{k}=\sum_{i=1}^{n} H\left(u_{i}, \hat{\theta}\right)$. The distribution of this test statistic $Y_{n}^{2}$ is chi-square (see Voinov et al. (2013)).

\subsection{Criteria test for $U G I W D$}

For testing the null hypothesis $H_{0}$ that data belong to the $U G I W$ model, we construct a modified chi-squared type goodness-of-fit test based on the statistic $Y^{2}$. Suppose that $\tau$ is a finite time and the observed data are grouped into $k>s$ sub-intervals $I_{j}=\left(a_{j-1}, a_{j}\right]$ of $[0, \tau]$. The limit intervals $a_{j}$ are considered as random variables such that the expected numbers of failures in each interval $I_{j}$ are the same, so the expected numbers of failures $e_{j}$ are obtained as

$$
E_{j}=\frac{-j}{k-1} \sum_{i=1}^{n} \ln \left(1-e^{-\alpha u_{i}^{\beta}}\right), \quad j=1, . . k-1
$$

and

$$
\hat{a}_{j}=\left[\frac{1}{\lambda}\left(-\frac{1}{\alpha} \ln \left(1-\exp \left(\frac{E_{j}+\sum_{l=1}^{i-1} \ln \left(1-e^{-\alpha u_{l}^{\beta}}\right)}{n-i+1}\right)\right)\right)^{1 / \beta}+1\right]^{-1}, \quad j=1, . . k-1
$$




\section{Estimated matrix $\hat{W}$}

The components of the estimated matrix $\hat{W}$ are derived from the estimated matrix $\hat{C}$ which is given by:

$$
\begin{aligned}
\hat{C}_{1 j} & =\frac{1}{n} \sum_{i: x_{i} \in I_{j}}^{n} \Delta_{i}\left[\frac{1}{\alpha}-\frac{\left(\lambda u_{i}\right)^{\beta}}{1-e^{-\alpha\left(\lambda u_{i}\right)^{\beta}}}\right] \\
\hat{C}_{2 j} & =\frac{1}{n} \sum_{i: x_{i} \in I_{j}}^{n} \Delta_{i}\left[\frac{\beta}{\lambda}-\frac{\alpha \beta \lambda^{\beta-1} u_{i}^{\beta}}{1-e^{-\alpha\left(\lambda u_{i}\right)^{\beta}}}\right] \\
\hat{C}_{3 j} & =\frac{1}{n} \sum_{i: x_{i} \in I_{j}}^{n} \Delta_{i}\left[\frac{1}{\beta}+\ln \lambda u_{i}-\frac{\alpha\left(\lambda u_{i}\right)^{\beta} \ln \left(\lambda u_{i}\right)}{1-e^{-\alpha\left(\lambda u_{i}\right)^{\beta}}}\right]
\end{aligned}
$$

and

$$
\hat{W}_{l}=\sum_{j=1}^{k} \hat{C}_{l j} A_{j}^{-1} Z_{j}, \quad l=1, . ., m \quad j=1, . ., k .
$$

Therefore, the quadratic form of the test statistic can be obtained easily as:

$$
Y_{n}^{2}(\hat{\theta})=\sum_{j=1}^{k} \frac{\left(U_{j}-e_{j}\right)^{2}}{U_{j}}+\hat{W}^{T}\left[\hat{\imath}_{l l^{\prime}}-\sum_{j=1}^{k} \hat{C}_{l j} \hat{C}_{l^{\prime} j} \hat{A}_{j}^{-1}\right]^{-1} \hat{W}
$$

\section{Simulation results for censored data}

We generated $N=10,000$ right censored samples with different sample sizes and different parameter values from the $U G I W$ model. Using $R$ statistical software and the Barzilai-Borwein $(B B)$ algorithm (Ravi and Gilbert 2009), we calculate the averages of the simulated values of maximum likelihood estimates of the unknown parameters and their corresponding mean squared errors (MSEs). The results are presented in Table 10. From Table 10, we can notice that the mean squared errors are very small, which confirms the convergence of the maximum likelihood estimators.

Table 10: Averages of the simulated values of MLEs $\hat{\alpha}, \hat{\lambda}$ and $\hat{\beta}$ and their corresponding mean squared errors

\begin{tabular}{clllllc}
\hline$N=10,000$ & $n_{1}=15$ & $n_{2}=25$ & $n_{3}=50$ & $n_{4}=130$ & $n_{5}=350$ & $n_{6}=500$ \\
\hline$\hat{\alpha}=1.2$ & $1.1398(0.0154)$ & $1.1521(0.0126)$ & $1.1623(0.0094)$ & $1.1756(0.0072)$ & $1.1934(0.0046)$ & $1.2009(0.0023)$ \\
$\hat{\lambda}=0.9$ & $0.9374(0.0079)$ & $0.9326(0.0067)$ & $0.9299(0.0052)$ & $0.9212(0.0039)$ & $0.9156(0.0027)$ & $0.9010(0.0016)$ \\
$\hat{\beta}=3$ & $2.9384(0.0069)$ & $2.9541(0.0058)$ & $2.9613(0.0046)$ & $2.9765(0.0034)$ & $2.9821(0.0018)$ & $2.9987(0.0009)$ \\
\hline & $n_{1}=15$ & $n_{2}=25$ & $n_{3}=50$ & $n_{4}=130$ & $n_{5}=350$ & $n_{6}=500$ \\
\hline$\hat{\alpha}=2.7$ & $2.6621(0.0106)$ & $2.6689(0.0082)$ & $2.6776(0.0061)$ & $2.6897(0.0047)$ & $2.6933(0.0032)$ & $2.6995(0.0012)$ \\
$\hat{\lambda}=1.5$ & $1.5386(0.0112)$ & $1.5314(0.0091)$ & $1.5263(0.0079)$ & $1.5195(0.0068)$ & $1.5128(0.0053)$ & $1.5012(0.0033)$ \\
$\hat{\beta}=2$ & $1.9587(0.0082)$ & $1.9624(0.0065)$ & $1.9765(0.0041)$ & $1.9875(0.0025)$ & $1.9902(0.0016)$ & $1.9999(0.0007)$ \\
\hline & $n_{1}=15$ & $n_{2}=25$ & $n_{3}=50$ & $n_{4}=130$ & $n_{5}=350$ & $n_{6}=500$ \\
\hline$\hat{\alpha}=3$ & $3.0421(0.0089)$ & $3.0374(0.0076)$ & $3.0246(0.0051)$ & $3.0108(0.0034)$ & $3.0034(0.0013)$ & $3.0003(0.0005)$ \\
$\hat{\lambda}=1.8$ & $1.8462(0.0074)$ & $1.8345(0.0055)$ & $1.8204(0.0038)$ & $1.8124(0.0021)$ & $1.8026(0.0012)$ & $1.8001(0.0003)$ \\
$\hat{\beta}=0.7$ & $0.6612(0.0097)$ & $0.6694(0.0086)$ & $0.6748(0.0067)$ & $0.6823(0.0046)$ & $0.6920(0.0037)$ & $0.6998(0.0017)$ \\
\hline
\end{tabular}

\subsection{Simulation results for test statistic $Y^{2}$}

In order to study the performance of the test statistic proposed in this work, a simulation study has been carried out. Thus, for testing the null hypothesis $H_{0}$ with respect to sample belongs to $U G I W$ distribution, we draw 10,000 samples data from $U G I W$ model with different sample 
sizes and different parameter values to calculate $Y^{2}$ statistic. Then, we compute the number of cases of rejection of the null hypothesis $H_{0}$, when the values of criteria statistic $Y^{2}$ are superior to $\chi_{\epsilon}^{2}(k)$ (the quantile of the chi-square distribution with $k$ degrees of freedom). We give a comparison between the different theoretical values of significance level $\epsilon$ (with $\epsilon=0.10, \epsilon=0.05, \epsilon=0.01$ ) and their simulated levels (empirical levels) of significance in Table 11. As can be seen, the values of the calculated empirical levels of $Y^{2}$ test are very close to those of their corresponding theoretical levels of the chi-squared distributions with $k$ degrees of freedom. Thus, we conclude that the proposed test is well suited to the UGIW distribution.

Table 11: Simulated levels of significance for $Y_{n}^{2}$ test for UGIW $(\hat{\theta})$ model against their theoretical values $(\epsilon=0.01,0.05,0.10)$

\begin{tabular}{llllllc}
\hline $\mathrm{N}=10,000$ & $n_{1}=10$ & $n_{2}=20$ & $n_{3}=50$ & $n_{4}=130$ & $n_{5}=350$ & $n_{6}=500$ \\
\hline$\epsilon=1 \%$ & 0.0063 & 0.0072 & 0.0085 & 0.0092 & 0.0095 & 0.0106 \\
$\epsilon=5 \%$ & 0.0321 & 0.0336 & 0.0398 & 0.0412 & 0.0475 & 0.0496 \\
$\epsilon=10 \%$ & 0.0846 & 0.0874 & 0.0890 & 0.0902 & 0.0951 & 0.0989 \\
\hline$N=10,000$ & $n_{1}=10$ & $n_{2}=20$ & $n_{3}=50$ & $n_{4}=130$ & $n_{5}=350$ & $n_{6}=500$ \\
\hline$\epsilon=1 \%$ & 0.0046 & 0.0053 & 0.0064 & 0.0072 & 0.0081 & 0.0092 \\
$\epsilon=5 \%$ & 0.0442 & 0.0467 & 0.0471 & 0.0483 & 0.0494 & 0.0509 \\
$\epsilon=10 \%$ & 0.0916 & 0.0942 & 0.0962 & 0.0974 & 0.0989 & 0.1005 \\
\hline$N=10,000$ & $n_{1}=10$ & $n_{2}=20$ & $n_{3}=50$ & $n_{4}=130$ & $n_{5}=350$ & $n_{6}=500$ \\
\hline$\epsilon=1 \%$ & 0.0043 & 0.0055 & 0.0066 & 0.0077 & 0.0086 & 0.0095 \\
$\epsilon=5 \%$ & 0.0375 & 0.0394 & 0.0428 & 0.0451 & 0.0465 & 0.0489 \\
$\epsilon=10 \%$ & 0.0829 & 0.0862 & 0.0898 & 0.0924 & 0.0945 & 0.0978 \\
\hline
\end{tabular}

\section{Application to right censored real data}

We consider the following data consisting of 42 pieces of strength of a certain type of braided cord that had been weathered for a specified length of time. This data set is taken from Crowder, Kimber, Smith, and Sweeting (1991). The observed right-censored strength-values are given below:

$26.8^{*}, 29.6^{*}, 33.4^{*}, 35^{*}, 36.3,40^{*}, 41.7,41.9^{*}, 42.5^{*}, 43.9,49.9,50.1,50.8,51.9,52.1,52.3,52.3,52.4$, $52.6,52.7,53.1,53.6,53.6,53.9,53.9,54.1,54.6,54.8,54.8,55.1,55.4,55.9,56,56.1,56.5,56.9$, $57.1,57.1,57.3,57.7,57.8,58.1,58.9,59,59.1,59.6,60.4,60.7$

We divide the data by 60.7 in order to get data lies between 0 and 1 .

We use the test statistic provided above to verify whether the above data set can be modeled by $U G I W$ distribution, and at this end, we first calculate the maximum likelihood estimators of the unknown parameters

$$
\hat{\theta}=(\hat{\alpha}, \hat{\lambda}, \hat{\beta})^{T}=(2.1865,5.623,3.2152)^{T}
$$

To calculate the test statistic $Y_{n}^{2}$, we need the following results (see Table 12). We choose $k=5$ grouping intervals of $I_{j}$.

So, we obtain the value of $Y_{n}^{2}$ as

$$
Y_{n}^{2}=X^{2}+Q=4.1387+1.9568=6.0955
$$

For significance level $\varepsilon=0.05$, the critical value $\chi_{5}^{2}=11.0705$ is greater than the value of $Y_{n}^{2}=6.0955$, so we can say that the proposed model $U G I W$ fit these data. 
Table 12: Values of $\widehat{a}_{j}, e_{j}, U_{j}, \hat{C}_{1 j}, \hat{C}_{2 j}, \hat{C}_{3 j}$

\begin{tabular}{|l|l|l|l|l|l|}
\hline$\hat{a}_{j}$ & 0.7085 & 0.8715 & 0.9099 & 0.9422 & 1 \\
\hline$U_{J}$ & 9 & 11 & 10 & 8 & 10 \\
\hline$\hat{C}_{1 j}$ & -0.6358 & -0.8459 & -0.6274 & -0.8452 & -0.7965 \\
\hline$\hat{C}_{2 j}$ & -0.8647 & -0.9658 & -1.1526 & 0.0254 & 0.0845 \\
\hline$\hat{C}_{3 j}$ & 0.8596 & 0.9485 & 0.8124 & 0.7485 & 0.8174 \\
\hline$e_{j}$ & 1.3859 & 1.3859 & 1.3859 & 1.3859 & 1.3859 \\
\hline
\end{tabular}

We also calculated the test statistic $Y_{n}^{2}$ to fit the data set to the competing models. The results are given in Table 13 .

Table 13: Values of the test statistic $Y_{n}^{2}$ for strength of a braided cord data of different competing models

\begin{tabular}{|l|l|}
\hline Modeling distribution & $Y_{n}^{2}$ \\
\hline$U G I W D$ & 6.0955 \\
\hline$U B S$ & 8.2635 \\
\hline$U G$ & 6.4253 \\
\hline$U W$ & 6.9568 \\
\hline$K W$ & 7.6352 \\
\hline$U n i t-$ BurII & 7.8569 \\
\hline
\end{tabular}

\section{Concluding remarks}

In this study, a new bounded distribution has been introduced in the $(0,1)$ intervals by transformation method which provides better fits than unit-Birnbaum-Saunders, unit-Weibull, Unit-Gompertz, Unit Burr-III and Kumaraswamy distributions. Some statistical properties has been derived. The unknown parameters of the UGIW distribution are estimated by six different frequentist methods of estimation and obtained their CIs. The practical applicability of the UGIW distribution has been illustrated by means of one real-life data application. Next, we provide the formulae of the criteria statistic of the modified chi-squared goodness-of-fit test for UGIW model when data are right censored and the parameters are unknown. The statistic $Y^{2}$ can be used to check the validity of the UGIW model. The main advantage of the chi-square goodness-of-fit tests for censored data is that the limiting distribution of these statistics is the well-known $\chi^{2}$ distribution. We hope that the results obtained through this study will be useful for practionnars in several fields. The performances of the results and the effectiveness of the proposed test are shown by simulation study and real data analysis.

\section{Acknowledgments}

The authors would like to thank the Editor, Associate Editor and the referees for careful reading and for valuable comments that greatly improved the presentation of article.

\section{Conflict of interest}

On behalf of all authors, the corresponding author states that there is no conflict of interest. 


\section{References}

Abd-Elfattah AM (2011). "Goodness of Fit Test for the Generalized Rayleigh Distribution." Journal of Statistical Computation and Simulation, 83, 357-366.

Abdelfattah EH (2008). "Goodness of Fit Tests for the Two Parameter Weibull Distribution." Journal of Statistical Theory and Applications, 7, 279-291.

Aidi K, Seddik-Ameur N (2016). "Chi-square Tests for Generalized Exponential AFT Distributions with Censored Data." Electronic Journal of Applied Statistical Analysis, 9, 371-384.

Al-Omari AI, Zamanzade E (2016). "Different Goodness of Fit Tests for Rayleigh Distribution in Ranked Set Sampling." Pakistan Journal of Statistics and Operation Research, XII, 2539.

Aryal G, Elbatal I (2015). "Kumaraswamy Modified Inverse Weibull Distribution." Applied Mathematics and Information Sciences, 9, 651-660.

Badr MM (2019). "Goodness-of-fit Tests for the Compound Rayleigh Distribution with Application to Real Data." Heliyon. doi:https://doi .org/10.1016/j.heliyon.2019.e02225.

Bagdonavičius V, Levuliene RJ, Nikulin M (2013). "Chi-squared Goodness-of-fit Tests for Parametric Accelerated Failure Time Models." Communications in Statistics-Theory and Methods, 42, 2768-2785.

Bagdonavičius V, Nikulin M (2011). "Chi-squared Goodness-of-fit Test for Right Censored Data." International Journal of Applied Mathematics and Statistics, 24, 30-50.

Balakrishnan N, Basu AP (1995). The Exponential Distribution: Theory, Methods and Applications. Gordon and Breach Publishers, Amsterdam.

Basheer AM (2019). "Alpha Power Inverse Weibull Distribution with Reliability Application." Journal of Taibah University of Science, 13, 423-432.

Chandra M, Singpurwalla ND, Stephens MA (1981). "Kolmogorov Statistics for Tests of Fit for the Extreme-value and Weibull Distributions." Journal of the American Statistical Association, 76, 729-731.

Cheng RCH, Amin N (1983). "Estimating Parameters in Continuous Univariate Distributions with a Shifted Origin." Royal Statistical Society Series B (Statistical Methodology), 45, 394403.

Chouia S, Seddik-Ameur N (2017). "A Modified Chi-square Test for Bertholon Model with Censored Data." Communication in Statistics- Simulation and Computation, 46, 593-602.

Crowder MJ, Kimber AC, Smith RL, Sweeting TJ (1991). Statistical Analysis of Reliability Data. CHAPMAN \& HALL/CRC.

De Gusmao FRS, Ortega EMM, Cordeiro GM (2011). "The Generalized Inverse Weibull Distribution." Statistical Papers, 52, 591-619.

Durbin J (1975). "Kolmogorov-Smirnov Tests when Parameters Are Estimated with Applications to Tests of Exponentially and Test on Spacings." Biometrica, 62, 5-22.

Elbatal I, Condino F, Domma F (2016). "Reflected Generalized Beta Inverse Weibull Distribution: Definition and Properties." Sankhya, 78, 316-340.

Gomez-Deniz E, Sordo MA, Caldern-Ojeda E (2013). "The Log-Lindley Distribution as an Alternative to the Beta Regression Model with Applications in Insurance." Insurance: Mathematics and Economics, 54, 49 -57. 
Goual H, Yousof HM (2020). "Validation of Burr XII Inverse Rayleigh Model via a Modified Chi-squared Goodness-of-fit Test." Journal of Applied Statistics, 47, 393-423.

Gradshteyn IS, Ryzhik IM (2014). Table of Integrals, Series, and Products. Sixth edition. San Diego: Academic Press.

Green JR, Hegazy YAS (1976). "Powerful Modified EDF Goodness-of-fit Tests." Journal of the American Statistical Association, 71, 204-209.

Hanook S, Shahbaz MQ, Mohsin M, Kibria BMG (2013). "A Note on Beta Inverse Weibull Distribution." Communications in Statistics - Theory and Methods, 42, 320-335.

Hassan AS (2005). "Goodness-of-fit for the Generalized Exponential Distribution." InterStat, 19, $1-15$.

Kao JHK (1958). "Computer Methods for Estimating Weibull Parameters in Reliability Studies." IRE Transactions on Reliability and Quality Control, 13, 15-22.

Khan MS, King R (2012). "Modified Inverse Weibull Distribution." Statistics and Applied Probability, 1, 115-132.

Kumaraswamy P (1980). "A Generalized Probability Density Function for Double Bounded Random Processes." Journal of Hydrology, 46, 79-88.

Mazucheli J, Menezes AFB, Dey S (2018b). "Improved Maximum-likelihood Estimators for the Parameters of the Unit-gamma Distribution." Communications in Statistics-Theory and Methods, 47, 3767-3778.

Mazucheli J, Menezes AFB, Dey S (2018c). "The Unit-Birnbaum-Saunders Distribution with Applications." Chilean Journal of Statistics, 9, 47-57.

Mazucheli J, Menezes AFB, Dey S (2019). "Unit-Gompertz Distribution with Applications." STATISTICA, LXXIX, 25-43.

Mazucheli J, Menezes AFB, Ghitany ME (2018a). "The Unit-Weibull Distribution and Associated Inference." Journal of Applied Probability and Statistics, 13, 1-22.

Mudasir S, Ahmad SP (2018). "Weighted Version of Generalized Inverse Weibull Distribution." Journal of Modern Applied Statistical Methods, 17. doi:10.22237/jmasm/1555506264.

Murthy DNP, Xie M, Jiang R (2004). Weibull Models. Wiley, New Jersey.

Nair NU, Sankaran P, Balakrishnan N (2013). Quantile-based Reliability Analysis. Statistics for Industry and Technology. Springer, New York.

Okasha HM, El-Baz AH, Tarabia AMK, Basheer AM (2017). "Extended Inverse Weibull Distribution with Reliability Application." Journal of the Egyptian Mathematical Society, 25, 343-349.

Pararai M, Warahena G, Oluyede BO (2014). "A New Class of Generalized Inverse Weibull Distribution with Applications." Journal of Applied Mathematics \& Bioinformatics, 04, $17-35$.

Ravi V, Gilbert PD (2009). "An R Package for Solving a Large System of Nonlinear Equations and for Optimizing a High-dimensional Nonlinear Objective Function." Journal of Statistical Software, 32, 1-26.

Seddik-Ameur N, Wafa T (2018). "On Testing the Fit of Accelerated Failure Time and Proportional Hazard Weibull Extension Models." Journal of Statistical Theory and Practice, 12, 397-411. 
Smith RL, Naylor JC (1987). "A Comparison of Maximum Likelihood and Bayesian Estimators for the Three-parameter Weibull Distribution." Journal of Applied Statistics, 36, $358-369$.

Stephens MA (1970). "Use of Kolmogorov-Smirnov, Cramer-von Mises and Related Statistics without Extensive Tables." Journal of the Royal Statistical Society, B(32), 115-122.

Stephens MA (1974). "EDF Statistics for Goodness of Fit and Some Comparisons." Journal of the American Statistical Association, 69, 730-737.

Swain J, Venkatraman S, Wilson J (1988). "Least Squares Estimation of Distribution Function in Johnson's Translation System." Journal of Statistical Computation and Simulation, 29, $271-297$.

Voinov V, Nikulin M, Balakrishnan N (2013). Chi-Squared Goodness of Fit Tests with Applications. Academic Press, Elsevier.

Wang B (2008). "Goodness-of-fit Test for the Exponential Distribution Based on Progressively Type-II Censored Sample." Journal of Statistical Computation and Simulation, 78, 125-132.

Yen VC, Moore AH (1988). "Modified Goodness-of-fit Test for the Laplace Distribution." Communication in Statistics- Simulation and Computation, 17, 275-281.

Zamanzade E, Mohdizadeh M (2017). "Goodness of Fit Tests for Rayleigh Distribution Based on Phi-divergence." Revistat Colmbiana de Estadistica, 40, 279-290.

\section{Appendix}

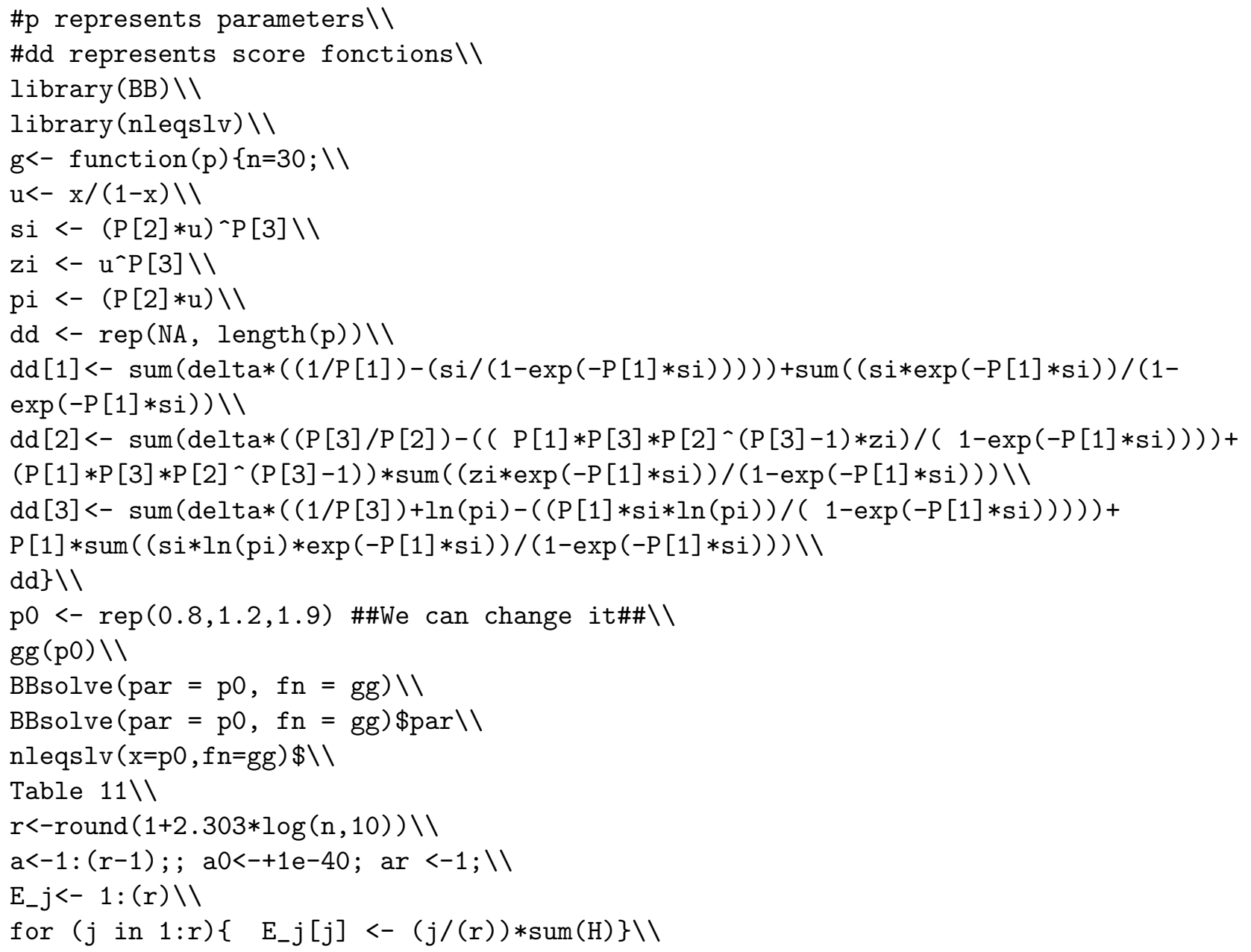




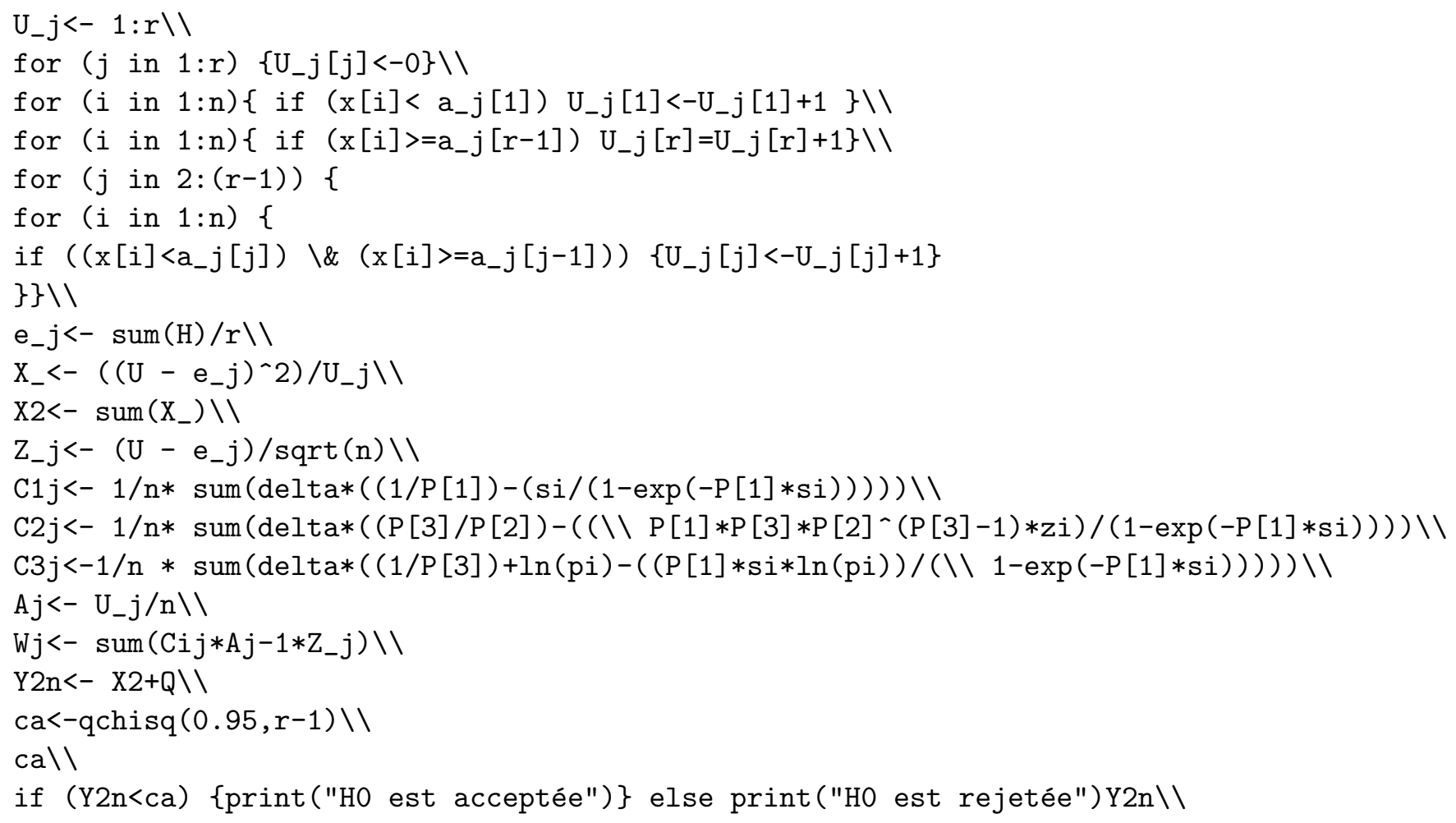

\section{Affiliation:}

Aidi khaoula

Laboratory of probability and statistics LaPS

University Badji Mokhtar-Annaba- Algeria

Sanku Dey

Department of Statistics

St. Anthony's College, Shillong-793001, Meghalaya, India

E-mail: sankud66@gmail.com

Devendra Kumar

Department of Statistics

Central University of Haryana, Haryana, India

E-mail: devendrastas@gmail.com

Seddik-Ameur N

Laboratory of probability and statistics LaPS

University Badji Mokhtar-Annaba- Algeria

\section{Austrian Journal of Statistics}

published by the Austrian Society of Statistics

Volume 50

July 2021 http://www.ajs.or.at/

http://www.osg.or.at/

Submitted: 2020-06-09

Accepted: 2020-11-12 